\title{
A WEAR MODEL FOR DIESEL ENGINE EXHAUST VALVES
}

November 24, 2009

\author{
Peter J. Blau
}

Materials Science and Technology Division

Oak Ridge National Laboratory 


DOCUMENT AVAILABILITY
Reports produced after January 1, 1996, are generally available free via the U.S. Department of
Energy (DOE) Information Bridge.
Web site http://www.osti.gov/bridge
Reports produced before January 1, 1996, may be purchased by members of the public from the
following source.
National Technical Information Service
5285 Port Royal Road
Springfield, VA 22161
Telephone 703-605-6000 (1-800-553-6847)
TDD 703-487-4639
Fax 703-605-6900
E-mail info @ntis.gov
Web site http://www.ntis.gov/support/ordernowabout.htm
Reports are available to DOE employees, DOE contractors, Energy Technology Data Exchange
(ETDE) representatives, and International Nuclear Information System (INIS) representatives from
the following source.
Office of Scientific and Technical Information
P.O. Box 62
Oak Ridge, TN 37831
Telephone 865-576-8401
Fax 865-576-5728
E-mail reports@ osti.gov
Web site http://www.0sti.gov/contact.html

This report was prepared as an account of work sponsored by an agency of the United States Government. Neither the United States Government nor any agency thereof, nor any of their employees, makes any warranty, express or implied, or assumes any legal liability or responsibility for the accuracy, completeness, or usefulness of any information, apparatus, product, or process disclosed, or represents that its use would not infringe privately owned rights. Reference herein to any specific commercial product, process, or service by trade name, trademark, manufacturer, or otherwise, does not necessarily constitute or imply its endorsement, recommendation, or favoring by the United States Government or any agency thereof. The views and opinions of authors expressed herein do not necessarily state or reflect those of the United States Government or any agency thereof. 
Materials Science and Technology Division

\section{A WEAR MODEL FOR DIESEL ENGINE EXHAUST VALVES}

Peter J. Blau

Date Published: October 2009

Prepared by

OAK RIDGE NATIONAL LABORATORY

Oak Ridge, Tennessee 37831-6283

managed by

UT-BATTELLE, LLC

for the

U.S. DEPARTMENT OF ENERGY

under contract DE-AC05-00OR22725 



\section{Executive Summary}

The work summarized here comprises the concluding effort of a multi-year project, funded by the U.S. Department of Energy, Office of Vehicle Technologies. It supports the development of a better understanding of advanced diesel engine designs in which enhanced power density, energy efficiency, and emissions control place increasing demands upon the durability of engine materials. Many kinds of metallic alloys are used in engines depending on the operating stresses, temperatures, and chemical environments. Exhaust valves, for example, are subjected to high temperatures and repetitive surface contacts that place demands on durability and frictional characteristics of the materials. Valves must continue to seal the combustion chamber properly for thousands of hours of cyclic engine operation and under varying operating conditions. It was the focus of this effort to understand the wear processes in the valve-seat area and to develop a model for the surface deformation and wear of that important interface. An annotated bibliography is provided to illustrate efforts to understand valve wear and to investigate the factors of engine operation that affect its severity and physical manifestation.

The project for which this modeling effort was the final task, involved construction of a hightemperature repetitive impact test system as well as basic tribology studies of the combined processes of mechanical wear plus oxidation at elevated temperatures. Several publications resulted from this work, and are cited in this report. The materials selected for the experimental work were highperformance alloys based on nickel and cobalt. In some cases, engine-tested exhaust valves were made available for wear analysis and to ensure that the modes of surface damage produced in experiments were simulative of service. New, production-grade exhaust valves were also used to prepare test specimens for experimental work along with the other alloy samples.

Wear analysis of valves and seats run for hundreds of hours in heavy-duty diesels provided insights into the kinds of complexity that the contact conditions in engines can produce, and suggested the physical basis for the current approach to modeling. The model presented here involves four terms, two representing the valve response and two for its mating seat material. The model's structure assumes that wear that takes place under a complex combination of plastic deformation, tangential shear, and oxidation. Tribolayers form, are removed, and may reform. Layer formation affects the friction forces in the interface, and in turn, the energy available to do work on the materials to cause wear. To provide friction data for the model at various temperatures, sliding contact experiments were conducted from 22 to $850^{\circ} \mathrm{C}$ in a pin-on-disk apparatus at ORNL.

In order to account for the behavior of different materials and engine designs, parameters in all four terms of the model can be adjusted to account for wear-in and incubation periods before the dominant wear processes evolve to their steady-state rates. For example, the deformation rate is assumed to be maximum during the early stages of operation, and then, due to material workhardening and the increase in nominal contact area (which reduces the load per unit area), decreases to a lower rate at long times. Conversely, the rate of abrasion increases with time or number of cycles due to the build-up of oxides and tribo-layers between contact surfaces. The competition between deformation and abrasion results in complex, non-linear behavior of material loss per cycle of operation. Furthermore, these factors are affected by valve design features, such as the angle of incline of the valve seat.

Several modeling scenarios are presented to demonstrate how the wear profile versus number of cycles changes in response to: (a) different relative abrasion rates of the seat and valve materials, (b) the friction coefficient as a function of temperature, (c) the relative deformation contribution of valve and seat materials, and (d) an interruption in the dominant wear process. 


\section{CONTENTS}

\section{Page}

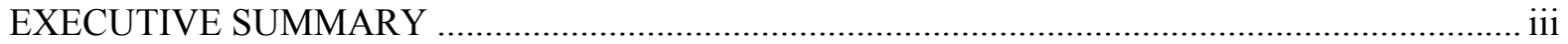

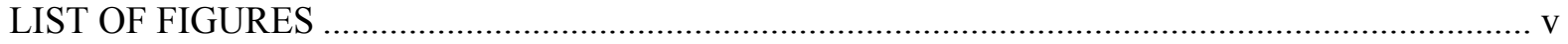

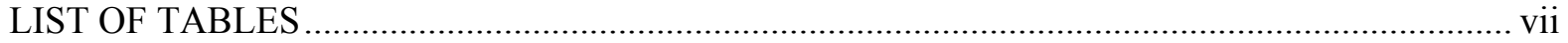

1 INTRODUCTION, BACKGROUND, AND PROJECT OBJECTIVES .................................... 1

2 FEATURES OF WORN EXHAUST VALVES …........................................................... 3

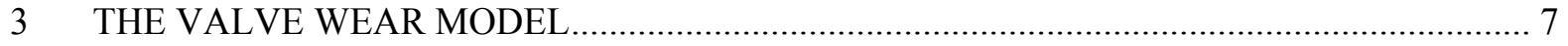

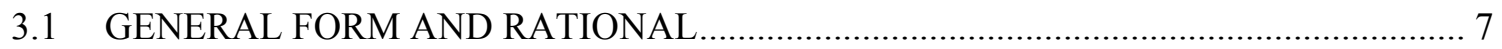

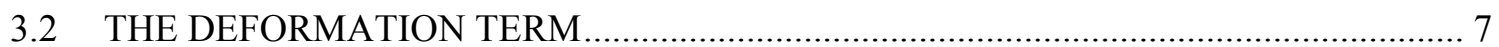

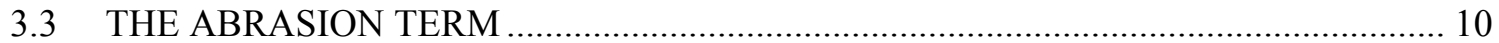

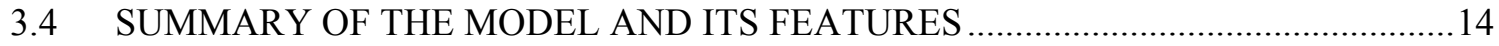

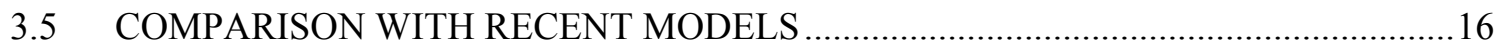

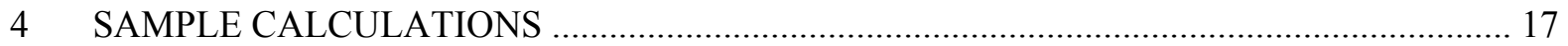

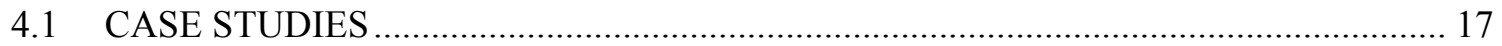

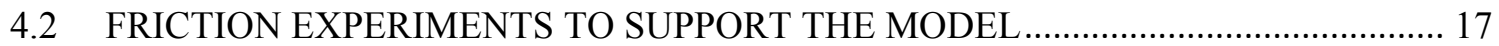

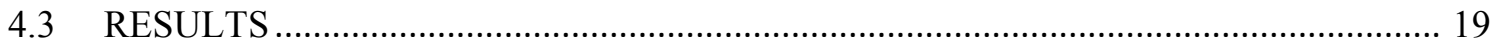

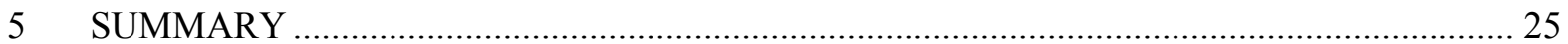

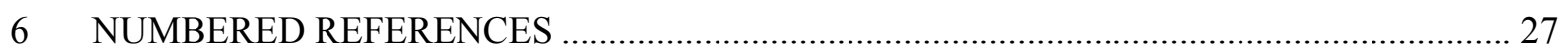

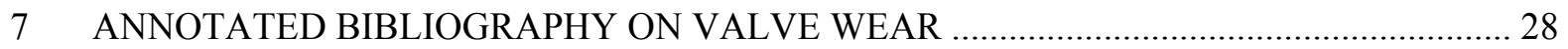





\section{LIST OF FIGURES}

$1 \quad$ Features of a typical exhaust valve .1

Schematic diagram of the contact between the valve and seat..........................................2

Schematic diagram of the High Temperature Repetitive Impact (HTRI apparatus .............2

Contact region on a worn exhaust valve showing wear features, oxides, and tribo-

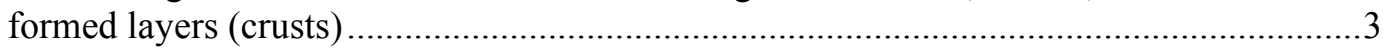

One area on an exhaust valve of the type shown in Figure 1 on which the metallic contact area bears the load. Oxide was prevented from remaining here due to the high deformation component and scrubbing action

Vertical scanning interferometric reconstruction of a worn face on a diesel engine exhaust valve. The view from lower left to upper right is from the stem up toward outside diameter of the valve tulip. In the color image, reddish areas are the highest features and blue areas lowest.

Secondary electron images of various locations on the sealing face of a used exhaust valve indicating a variety of features

Micro-cracking around a Knoop hardness impression in a tribolayer produced on

Ni-based, Pyromet $80 \mathrm{~A}$, created in the HTRI by 20,000 repetitive impacts from a Stellite $6 \mathrm{~B}$ counterface at $800^{\circ} \mathrm{C}$. Valid hardness numbers of tribolayers are difficult to obtain because of such micro-cracking

Seat from the same engine and exhaust valve as shown in Figures 5-7, but showing a very smooth, and nearly featurless appearance. The contact face is the wide, relatively featurless ban below of the bright circumferential grooves. The darker, mottled band above the bright grooves was not in contact.

Function depicting the decline in volume displaced per cycle after running-in..................8

Sum of incremental displacements (total sliding distance) arising from the

decreasing displacement rate function plotted in Figure 10

Transition from no slip per stroke to a steady-state value of slip per stroke after an incubation period of approximately 2000 cycles

Cumulative sliding distance after an initial period of minimal slip

14 High-temperature pin-on-disk sliding friction and wear testing system. The pin specimen holder is visible at the top entering the furnace chamber where a disk rotates against it

Sliding friction coefficient as a function of temperature for Stellite $6 \mathrm{~B}$ pin on a rotating disk of Pyromet 31

Area of the valve/seat profile due to the combined abrasive wear of both sides of the wear couple for Case 1 
17 Area of the valve/seat profile due to the combined abrasive wear of both sides of the wear couple for Case 1 except that the seat angle is decreased to 30 degress Plot of the area of the wear profile versus number of cycles of operation for the parameters applied in Case 1

Plot of the area of the wear profile versus number of cycles of operation for the parameters applied in Case 2

Plot of the area of the wear profile versus number of cycles of operation for the parameters applied in Case 3

Representation of a situation in which the wear rate is interrupted by the formation

of a protective tribolayer, then later resumes at its original steady-state rate ................... 24

Approximation of the worn profile as a rectangle of length $\mathrm{L}$

Estimation of valve stem recession from the model using Case 4 parameters ................. 25 



\section{LIST OF TABLES}

Table

Page

1 Summary of Nomenclature Used in the Valve Wear Model ...............................15

2 Summary of Nomenclature used in the Dwyer-Joyce Valve Recession

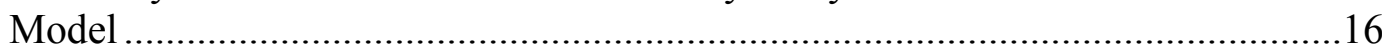

3 Summary of Variables Used to Test the Valve Wear Model...............................20 





\section{INTRODUCTION, BACKGROUND, AND PROJECT OBJECTIVES}

The majority of heavy trucks in the United States use diesel engines as the primary means of propulsion. Diesel engines are generally more efficient than spark ignition engines (reaching efficiencies of up to $40-45 \%$ ). Heavy trucks are estimated to consume about $20 \%$ of the on-highway fuels, amounting to over 33 Billion gallons per year in the U.S. [1]. Therefore, even small improvements in the energy efficiency of diesel engines can have a large and beneficial effect on the conservation of fossil fuels. At the same time, the minimization of harmful emissions is essential, so that even as the energy efficiency is improved, whatever combustion by-products are still produced will have less of an impact on the environment. Increasingly stringent emissions regulations have become a fact of life.

There are a number of technological opportunities to reduce diesel engine emissions and still improve their fuel economy. The U.S. Department of Energy has set ambitious goals to that end [2]. Some approaches involve changes in design, timing, and engine management. Others involve the application of new materials, surface treatments, or lubricants. Implementing these complex and interactive approaches has required increasingly sophisticated analytical tools and test methods. The current project focuses on the materials-related issue of exhaust value durability and lifetime performance. The objectives of the current project were to understand the processes of wear in diesel engine exhaust valves, study them experimentally, and develop a model to study the contributions of various physical variables on these processes.

The features of a typical exhaust valve are shown in Figure 1. The annular contact zone between the valve and the seat area of the insert the focus of this work and is shown schematically in Figure 2. The pursuit of the project objectives involved a three-pronged, multi-year approach: (a) studies of the effects of surface damage on oxidation of alloys, (b) the construction of an apparatus to study the response of metallic alloys under conditions of elevated temperature, oxidation, and repetitive impact with slip, and (c) the preparation of a model for valve wear, reported here. The apparatus that was built is called the High Temperature Repetitive Impact apparatus (HTRI) and it is shown in Figure 3.

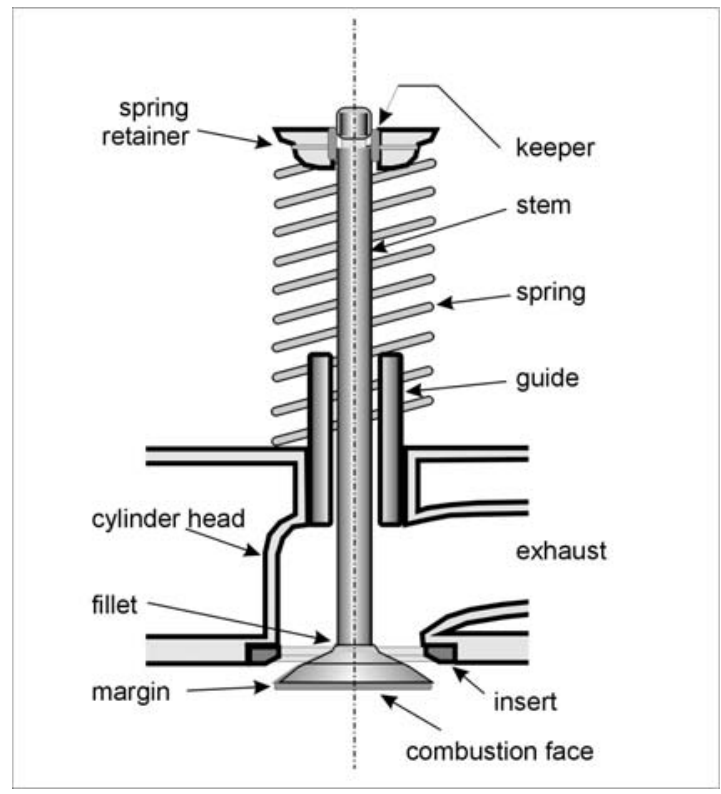

Figure 1. Features of a typical exhaust valve. 


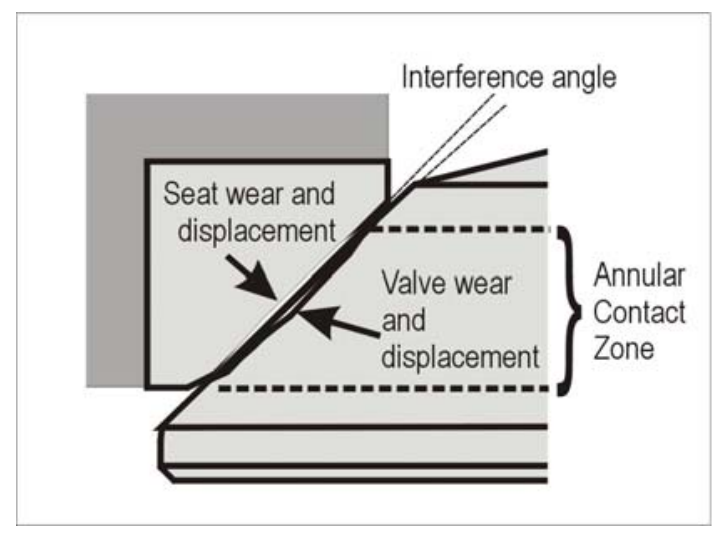

Figure 2. Schematic diagram of the contact between the valve and seat.

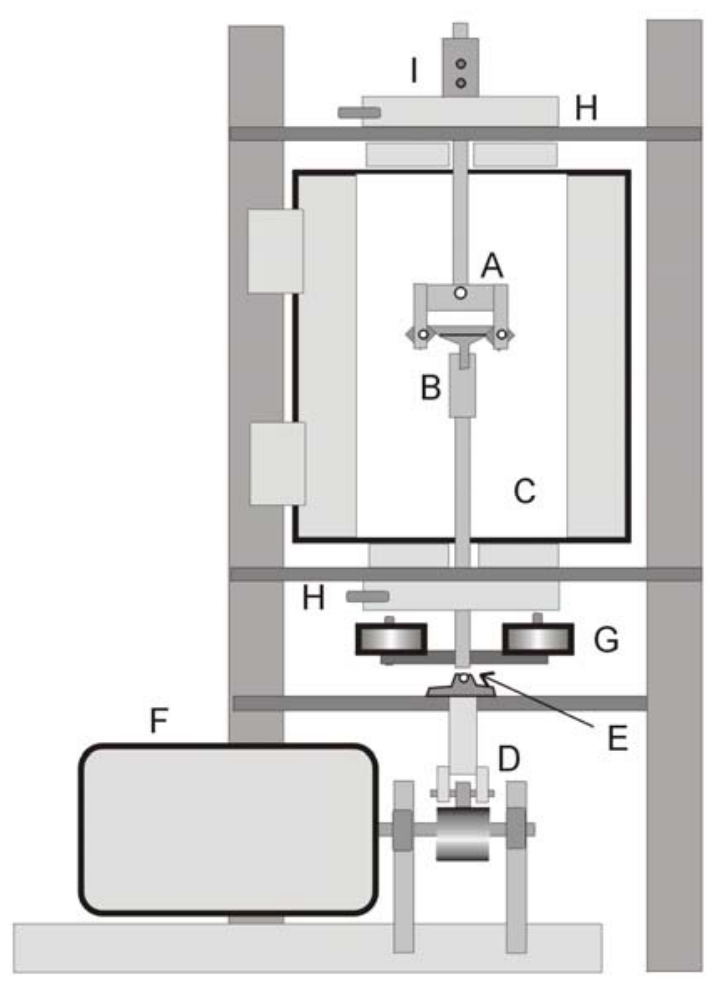

Figure 3. Schematic diagram of the High Temperature Repetitive Impact (HTRI) apparatus.

In parallel with HTRI experiments, studies were done on the role of wear when it occurs in conjunction with oxidation and at elevated temperatures [3-5]. It was found that once damaged by abrasion, a surface exposed to high temperature forms different oxide compositions than a surface that is exposed to high temperature, but not in an abraded condition. Scratch tests followed by heat treatments substantiated these effects.

The concept for building the HTRI was to provide repetitive impact on an angle such that the surfaces would experience a combination of normal impact plus slip, as does an exhaust valve striking the seat. That approach was similar to that taken earlier when designing a room-temperature testing apparatus (e.g., [6]). The former apparatus was used to study the effects of machining 
procedures on the durability of advanced ceramics for engine valves. The HTRI, however, was intended to better simulate actual exhaust valve operating conditions in a temperature range of $700^{\circ} \mathrm{C}$ to $850^{\circ} \mathrm{C}$ [7].

Concerning the mechanisms of valve wear, Giles [8] pointed out that larger seat angles and lower friction coefficients cause higher normal forces and more wedging between the valve face and insert. Higher normal forces tend to crush deposits that could otherwise build-up on the mating surfaces, but they also generate more wear. In cases of low-sulfur fuel, where the friction coefficients are somewhat higher, higher normal forces can be problematical. On the other hand, shallower seat angles reduce the shear stress and wear, but they allow deposits to form more readily. In the HTRI design, it was decided to use a standard seat angle of 45 degrees to emphasize the sliding component. In fact, specimens produced in the HTRI exhibited a compressed tribolayer very similar to those observed on worn valves from engines.

Exhaust valves and valve seats experience various modes of damage (See Ref [9] and Section 7.0). For example, valve face abrasion is caused by fine particles from residual deposits. Adhesive wear and micro-welding can occur to produce plastic shear and localized pull-out of surface material. Repetitive contact can result in plastic deformation and progressive lateral displacement. Oxidation also occurs on the mating surfaces, adding a tribochemical aspect to the wear process. The chemical composition of diesel engine exhaust gas (see, for example, Table 1) was not simulated in the present case; rather, the HTRI design focused on achieving high-temperature, repetitive impact with slip in addition to oxidizing conditions. Surfaces were examined after testing to determine the degree to which the common valve wear features were produced. The following section describes the surfaces of worn valves and highlights the complexity of their morphology and features.

\section{FEATURES OF WORN EXHAUST VALVES}

The surfaces of used exhaust valves display a variety of features, Figures 4 (a) and (b), for example, show two views of the contact surface of a heavy-duty diesel engine that was run for several hundred hours. It shows an annular contact band approximately $2 \mathrm{~mm}$ wide. The higher magnification image in Fig. 4(b) indicates a heterogeneous deposit with areas of oxides and combustion products (tribolayer). At the lower right, near the scale bar, a step can be seen at the edge of the contact due to repetitive impact and material loss. Figure 5 shows an area where the metal has been displaced and the oxide layer is below the level of the contact surface, allowing the metal contact surface to remain relatively bright.

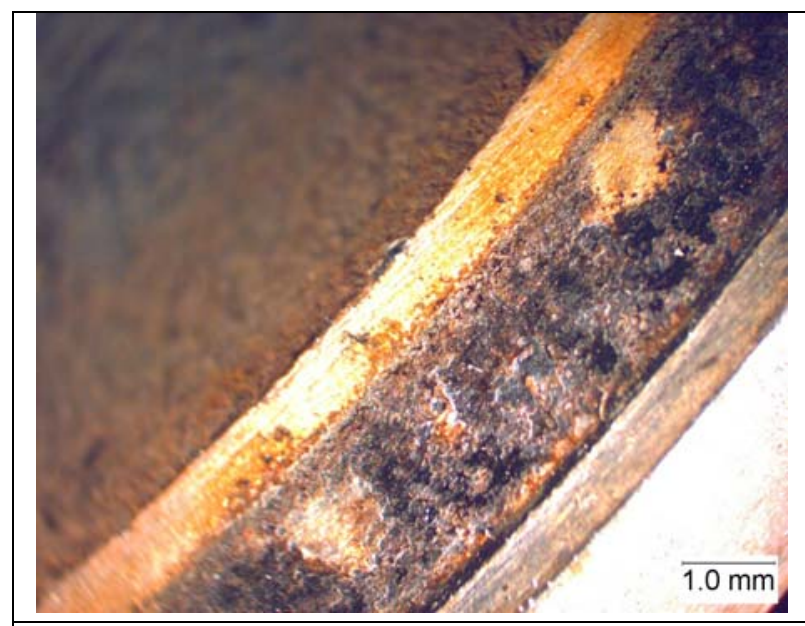

(a)

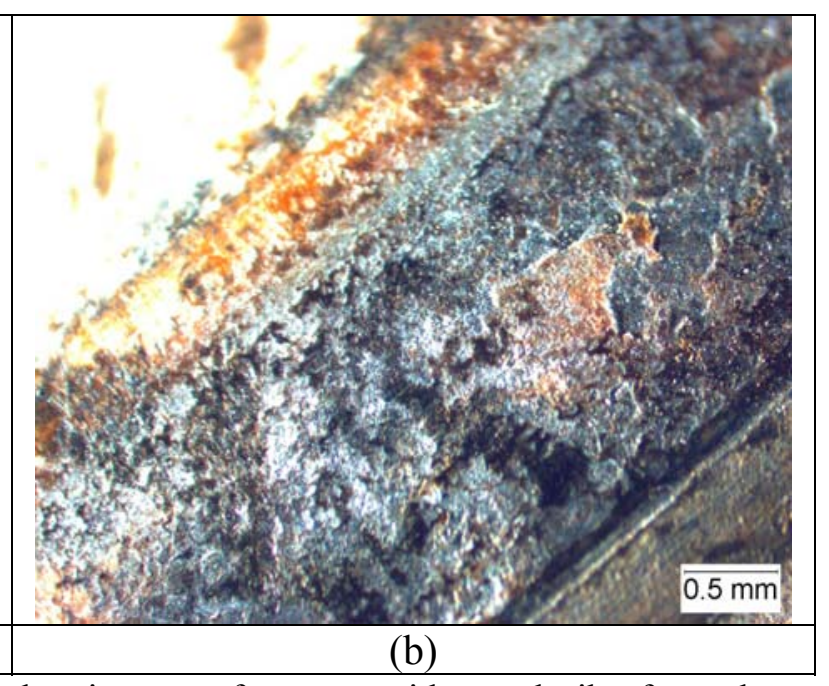

(b)

Figure 4. Contact region on a worn exhaust valve showing wear features, oxides, and tribo-formed layers (crusts). 


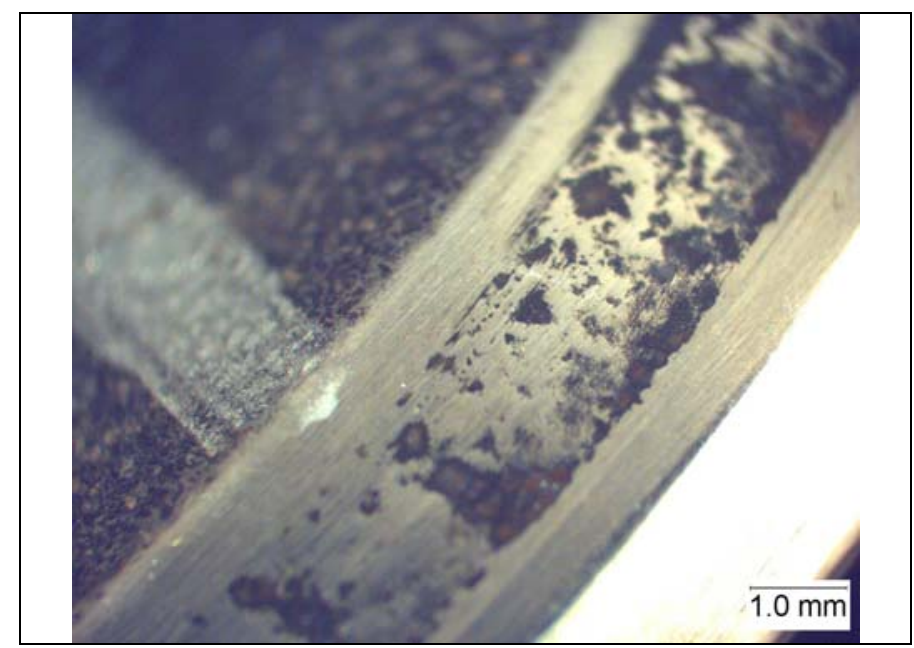

Figure 5. One area on an exhaust valve of the type shown in Figure 1 on which the metallic contact area bears the load. Oxide was prevented from remaining here due to the high deformation component and scrubbing action.

Figure 6 shows a three-dimensional image generated by a vertical scanning interferometer of a worn area such as that shown in Figure 4. Three-dimensional topography indicates lumpy areas of displaced material. High spots are in red, low in blue. Black areas are out of range of the instrument (Wyko NT9100 image). The apparent direction of view in Figure 6 (lower left to upper right) is from the top of the sealing face down and toward the outside diameter of the valve tulip. A clear step can be seen at the top of the image and bottom of the scan, where the sealing face has worn down.

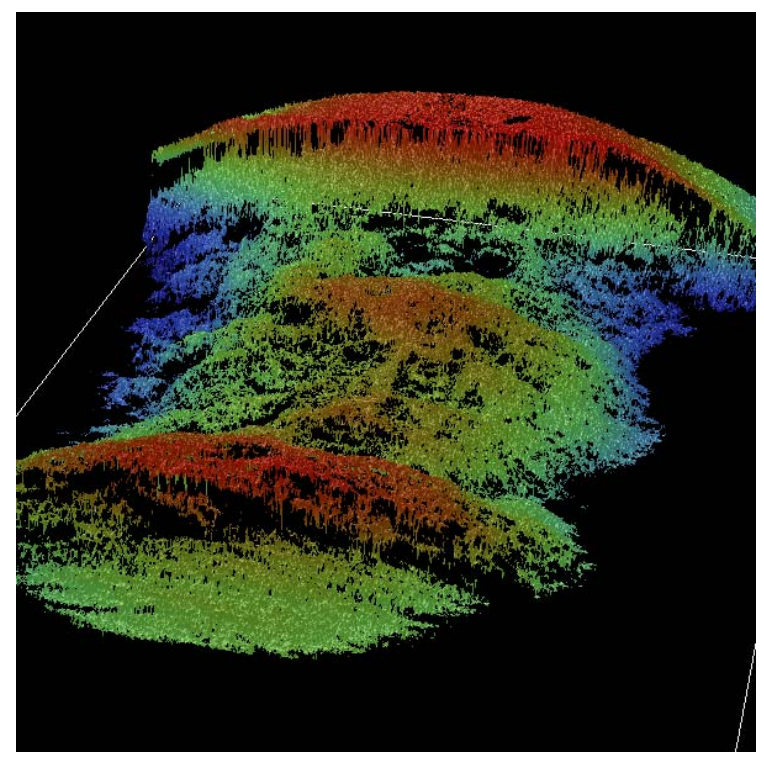

Figure 6. Vertical scanning interferometric reconstruction of a worn face on a diesel engine exhaust valve. The view from lower left to upper right is from the stem up toward outside diameter of the valve tulip. In the color image, reddish areas are the highest features and blue areas lowest. 
Scanning electron micrscopy was performed to study the nature of wear damage in more detail. Figures 7 (a) - (d) show some of the variety of features. The compositions, shapes, and mechanical characteristics of such features are not the same from place to place around the circumference of the sealing surfaces.

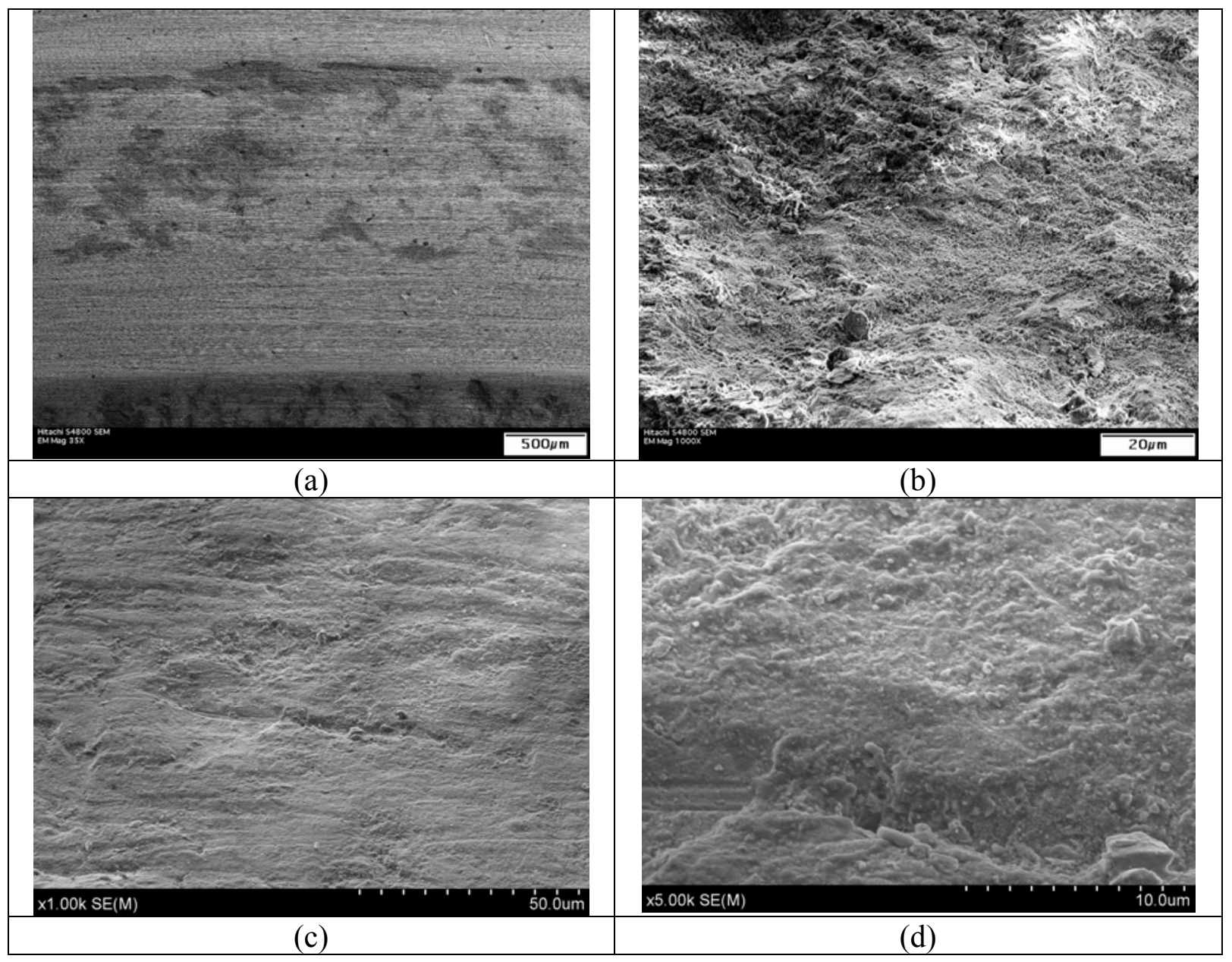

Figure 7. Secondary electron images of various locations on the sealing face of a used exhaust valve indicating a variety of features. (a) Area where the metal deformation was highest, and there was relatively little tribolayer. Oxides appear darker in this BSE image. (b) Another area on the same valve where compressed oxide debris resided. Bright areas are due to charging from non-conductive oxide deposits. (c) Smoother area of compressed debris showing light abrasion furrows in the circumferential direction, symptomatic of rotation of the valve about its central axis during use. (d) Higher magnification image of the central portion of (c) showing the sub-micron-sized grains that comprise the tribolayer.

Figure 8, from an HTRI experiment of Co-alloy impacting on Ni-alloy at $800^{\circ} \mathrm{C}$ (see Ref. [7]), indicates that tribolayers formed at high temperatures can be brittle, leading to micro-fracturing and spallation. In the experiment, contact motion was vertical to the image and there was no imposed circumferential sliding, as indicated by the horizontal grooves in Figure 7(c) and (d). However, the compressed nature of the metal/oxide layer produced by impact with low-amplitude slip is evident. 


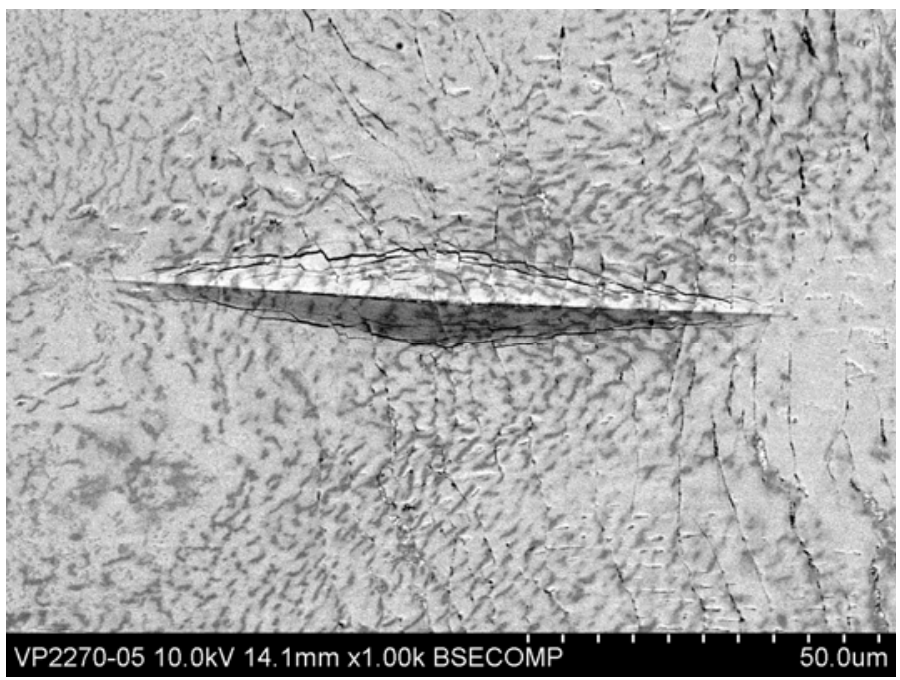

Figure 8. Micro-cracking around a Knoop hardness impression in a tribolayer produced on Ni-based, Pyromet $80 \mathrm{~A}$, created in the HTRI by 20,000 repetitive impacts from a Stellite $6 \mathrm{~B}$ counterface at $800^{\circ}$ C. Valid hardness numbers of tribolayers are difficult to obtain because of such micro-cracking. From Ref. [7].

Figure 9 shows a highly polished surface of a valve seat that rubbed against the valve shown in Figure 4. Compared to Figures 4 and 5 of the valve contact zone, the mating alloy did not display any of the gross wear features, but rather exhibited a very smooth, polished appearance, symptomatic of fine-scale abrasion.

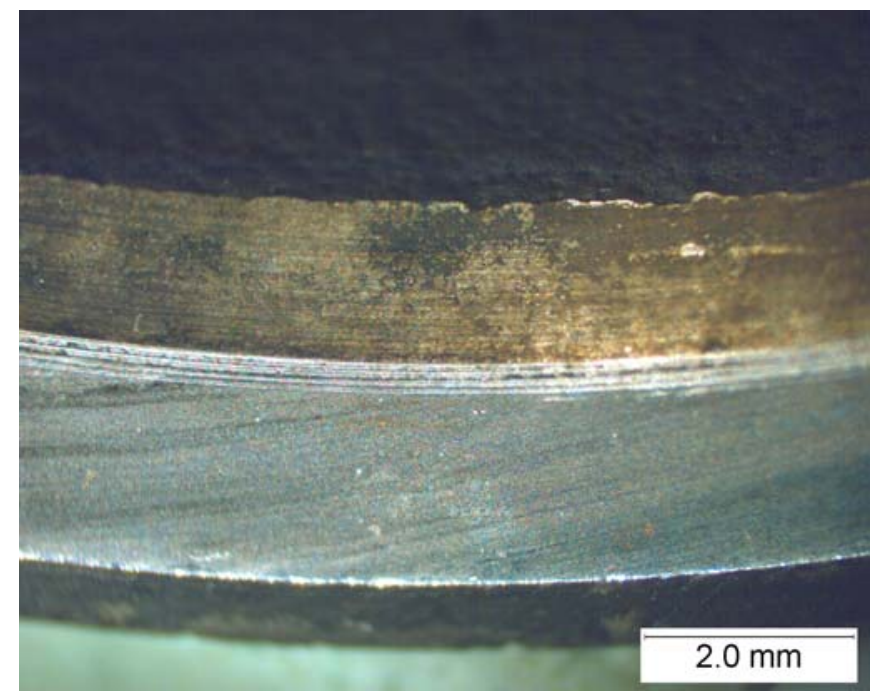

Figure 9. Seat from the same engine and exhaust valve as shown in Figures 5-7, but showing a very smooth, and nearly featureless appearance. The contact face is the wide, relatively featureless band below the bright circumferential grooves. The darker, mottled band above the bright grooves was not in contact.

Basic metallurgical principles indicate that upon exposure to high temperatures, metals and their alloys can change their properties and microstructures. For example, phase changes can occur, precipitates can ripen, grains can coarsen, atomic species with increased mobility can segregate to 
grain boundaries, or to the surface. In fact, it was shown that alloying additions and oxidation products in exhaust valve-like alloys of $\mathrm{Ni}$ and $\mathrm{Co}$ can redistribute differently over time at high temperature depending upon whether surfaces are mechanically-damaged [3-4]. Consequently, there is no inherent reason to expect that a steady-state rate of deformation or wear will be maintained as the interfacial materials age. As a result, the wear processes for exhaust valves and seats cannot be modeled as a simple linear function of time or number of number of impacts because the valve materials experience internal metallurgical changes due to aging, elemental diffusion, workhardening, and damage accumulation. Tribolayers of compressed debris form as well, and phenomena such as wear-in also occur.

Section 7.0 of this report provides an annotated bibliography of articles that concern the mechanisms of valve wear and the factors that affect them.

\section{THE VALVE WEAR MODEL}

\subsection{GENERAL FORM AND RATIONALE}

Rather than attempt to incorporate all of the foregoing surface damage mechanisms explicitly, it was decided to treat the valve wear model phenomenologically, producing a model that would allow one to examine the effects of changing parameters, like the angle of the valve seat, the relative abrasion rates of the seat and valve, and so forth. The approach taken here was to use what is known about wear phenomena on valves to calculate the effective cross-sectional area of the wear profile in the annular contact zone (shown schematically in Figure 2). Results of the model will therefore be presented in terms of removed area rather than as a volumetric loss rate or a change in the specific contact shape.

The general valve wear model consists of two contributions: one for displacement and one for abrasion. Both of these can contribute to the wear profile of the valve and seat combination.

Volumetric wear rates or displacement rates are converted into changes in the cross-sectional area of the valve-seat profile.

\subsection{THE DEFORMATION TERM}

The deformation term is based on the assumption that initial deformation occurs at the greatest rate (displacement per impact) and then gradually decreases to a smaller, steady-state value (or zero) after an initial running-in period. The volume of material displaced during a given impact cycle $\left(V_{\text {disp }}\right)$ can be represented using an exponential function of the type:

$$
f(x)=a \exp [b(x-c)]
$$

In terms of the number of cycles, $n$, and considering a constant post-run-in rate of deformation,

$$
g(n)=c_{\mathrm{d} 1} \exp \left[c_{\mathrm{d} 2}\left(n-c_{\mathrm{d} 3}\right)\right]+c_{\mathrm{d} 4}
$$

where $c_{\mathrm{d} 1}=$ the magnitude of the change between the initial displacement rate and the steady-state displacement rate of $c_{\mathrm{d} 4}, c_{\mathrm{d} 2}=$ a constant that reflects how quickly the displacement rate reaches its steady-state value, and $c_{\mathrm{d} 3}=$ the projected displacement per cycle at zero impacts. It is possible that the deformation rate will be negligible after running-in. In that case, $c_{\mathrm{d} 4} \sim 0$. An illustrative plot of the shape of this function is shown in Figure 10, where $\mathrm{g}(n)$ in essence represents $V_{\text {disp }}(\mathrm{n})$. 


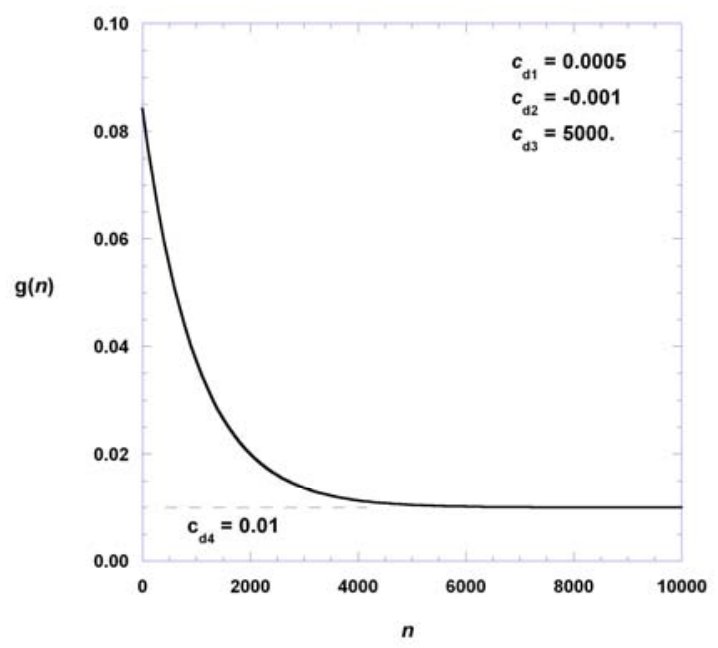

Figure 10. Function depicting the decline in volume displaced per cycle after running-in.

Physically, $c_{\mathrm{d} 1}$ and $c_{\mathrm{d} 2}$ reflect the effects of increasing contact area (i.e., decreasing nominal contact stress) as the mating surfaces conform during running-in. Furthermore, the magnitude of $c_{\mathrm{d} 1}$ is also related to the amount of work-hardening that occurs in the given material within the temperature range of operation. The more work-hardening, the less deformation there is per impact.

The cumulative volume displaced from a surface $\left(V_{\text {disp }}\right)$ is the sum of the individual displacements per cycle. Technically, one cycle involves two strokes (up and back), but deformation does not occur during the disengagement of surfaces, so the volume displaced is simply determined from the number of cycles.

$$
V_{\text {disp }}=\sum_{i=1}^{n} g(n)_{i}
$$

Using the decreasing deformation rate shown in Figure 10, the cumulative displacement is computed and the results are shown in Figure 11. If $c_{d 4}$ were zero, the plot in Figure 11, would level off to a constant value after about $\mathrm{n}=8000$. 


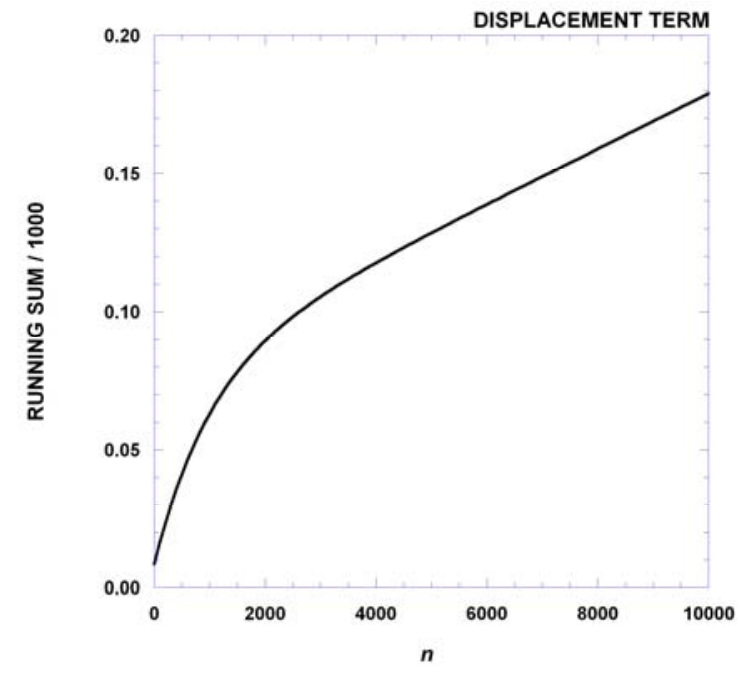

Figure 11. Sum of incremental displacements (total sliding distance) arising from the decreasing displacement rate function plotted in Figure 10.

The cross-sectional area of the displaced material on the plane that contains the incline of the valve or seat $\left(A_{\text {disp }}\right)$, can be calculated by dividing the cumulative displacement $V_{\text {disp }}$ by the average circumference of the ACZ (diameter $=D_{\text {acz }}$ ). Thus,

$$
A_{d i s p}=\frac{1}{\pi D_{a c z}} \sum_{i=1}^{n} f(n)_{i}
$$

The total cumulative displaced cross-sectional area for the valve and seat at a given number of impact cycles is then,

$$
\left.\left.A_{\text {disp }}(n)=A_{\text {disp }}(n)\right]_{\text {valve }}+A_{\text {disp }}(n)\right]_{\text {seat }}
$$

If displaced material in the ACZ moves out the contact zone, then the projected height of the displaced area for the interface, i.e., along the valve stem axis, $z$, would be externally observed as an increasing extension of the stem position when the valve is closed (seated).

Lip formation. If some of the material displaced were to form a lip on the 'uphill side' of the interface, the valve could be prevented from seating fully, and there could be a net reduction in the valve stem extension in the closed position. The change in valve extension $z$ due to wear can be expressed as the projection of the deformation that includes the formed lip $\left(\mathrm{z}_{\mathrm{lip}}\right)$ and a dish in the seating face $\left(\mathrm{z}_{\mathrm{dish}}\right)$,

$$
\Delta Z=Z_{\text {dish }}-Z_{\text {lip }}
$$

If the lip forms in a manner as to prevent seat closure, then $\Delta z$ may be negative, at least until the lip is worn off or further displaced until it does not interfere with seating. Lip formation, therefore, could be misinterpreted as 'negative wear' in external valve recession measurements. 
If deformation of the seat material and the valve material occur at different rates during normal engine operation, and momentarily neglecting the abrasion of the surfaces, the valve recession versus time will be non-linear. Further, if deformation is dominated by one or the other material, as when hard seats are used with lower-strength valves, this term can be simplified to include only the more deforming surface of the valve/seat tribo-couple.

A note on 'guttering.' In extreme cases, erosive gas leakage through the gaps produced by uneven circumferential seals may lead to a phenomenon called guttering in which channels develop on certain portions of the ACZ. Other sources of guttering include the build-up of localized deposits of ash, oil leaking through the valve guide onto the seating face, or from the caked products of incomplete combustion. Guttering was not included in the current model.

\subsection{THE ABRASION TERM.}

The second contribution to valve sealing surface wear is abrasion. Like other forms of wear, the cumulative abrasive wear volume for a surface, $V_{a b r}$, is related to materials properties, the conditions of contact, and the surrounding thermal and chemical environment. One can assume that the wear volume is proportional to the mechanical energy input to the surfaces. The mechanical energy available to do work in a sliding interface is proportional to the product of the friction force at the temperature of operation, $F_{T}$, and the cumulative sliding distance, $S(n)$. Therefore, a reasonable starting point to calculate the abrasive wear volume $V_{\text {abr }}$ is,

$$
V_{a b r}=k_{a b r} F S(n)
$$

where, $k_{a b r}=$ the abrasive wear rate characteristic of the material. The sliding distance per cycle is twice the distance slipped in the interface during each open/close event. That is because there is a short forward stroke upon seating and a short reverse stroke on withdrawal. The slip per cycle is not necessarily constant because, as the seating surfaces wear-in, the clearance may increase and allow the slip amplitude to increase as well. The length of slip per cycle $f(n)$ can be represented by a sigmoidally-behaving function such as the hyperbolic tangent:

$$
f(n)=a \tanh (x-b)
$$

where $a$ is a scaling factor for the magnitude of $f(x)$ and $b$ is the zero off-set of the mid-point of the transition to the maximum amplitude. In the present context, the slip as function of number of cycles

can be modeled as a process that begins with no slip initially, then it begins to increase until a steadystate value of the slip per cycle occurs. The introduction of an incubation period before substantial slip occurs accounts for the effect of deformation and dish formation before substantial slip occurs. The above equation is therefore modified as follows:

$$
f(n)=c_{a 1} \tanh \left[c_{a 2}\left(n-c_{a 3}\right)\right]+c_{a 1}=c_{a 1}\left\{\tanh \left[c_{a 2}\left(n-c_{a 3}\right)\right]+1\right\}
$$

where $c_{a 1}$ is one-half the magnitude of the steady-state slip length, $c_{a 2}$ is a rate constant for the transition from the initial to the final rate of slip per cycle, and $c_{a 3}$ is the number of cycles required to reach the mid-point of the transition from $n=0$ to the steady-state magnitude of slip (accounting for an incubation period). Figure 11 exemplifies the shape of this function for the parameters shown in the figure legend. 


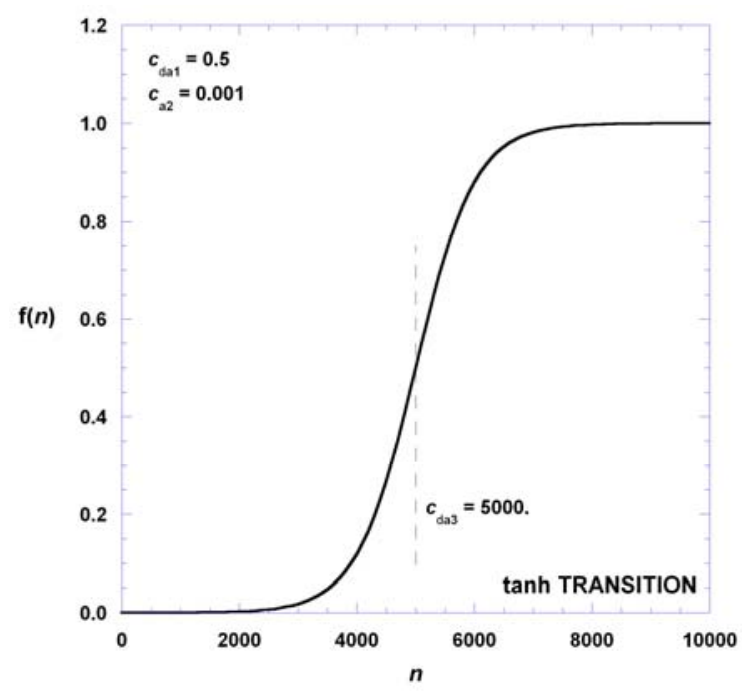

Figure 12. Transition from no slip per stroke to a steady-state value of slip per stroke after an incubation period of approximately 2000 cycles.

The slip during each cycle is summed to obtain a cumulative sliding distance $S(n)$. Since there are two strokes per cycle, the cumulative sliding distance for slip at the variable rate shown in Figure 12 can be calculated from

$$
S(n)=2 \sum_{i=1}^{n} f(i)
$$

. The results are illustrated in Figure 13.

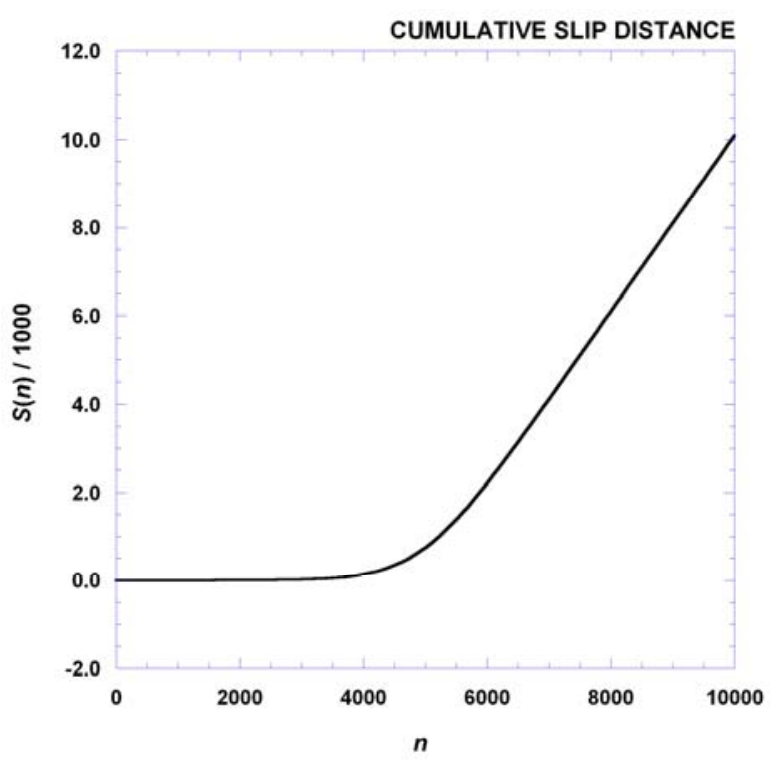

Figure 13. Cumulative sliding distance after an initial period of minimal slip. 
The normal force on the sliding interface is affected by the valve spring force and the combustion pressure. According to Schaeffer et al.[10], combustion pressure can exceed $18 \mathrm{MPa}(2611 \mathrm{psi})$. The combustion pressure $(p)$ is applied to the face of the exhaust valve having a typical diameter $(d)$ in the range of 40 to $45 \mathrm{~mm}$. Therefore, the force $P_{a x}$ in the axial direction, is

$$
P_{a x}=p A=p \pi\left(\frac{d}{2}\right)^{2}=0.7854 p d^{2}
$$

For a cylinder pressure of $18 \mathrm{MPa}$, and valve diameter of 40 to $45 \mathrm{~mm}, P_{a x}$ is in the range of 22.6 to $28.6 \mathrm{kN}$. The component of $P_{\mathrm{ax}}$ that is normal to the sliding interface in the ACZ, at 45 degrees, is

$$
P_{n}=P_{\text {ax }} \cos 45=0.7071 P_{a x}
$$

Consequently, the value of $P_{n}$ would be in the range of 16-20.2 kN More recent information about a typical heavy-duty diesel indicates a spring force of about $400 \mathrm{~N}$, and the normal force due to combustion echoes the aforementioned range of 15 to $20 \mathrm{kN}$ [11]. Based on the magnitudes involved, the combustion force is more likely to have a greater effect on the wear than the spring force.

It is known that the higher the seat angle, the greater is the sliding wear damage, and some engine makers are reconsidering that observation in light of the introduction of less lubricative, low-sulfur fuels. Furthermore, the energy available to do work on mating surfaces is proportional to the sliding friction coefficient $\mu$. From the definition of the friction coefficient $\left(\mu=F / P_{n}\right)$, the friction force arising from a pressure $p$, and at a given operating temperature $T$, is

$$
F_{T}=\mu_{T} p A_{\mathrm{v}} \cos \theta
$$

As mentioned above, the abrasive wear volume should in principle be related to the total frictional work expended over the sliding distance, viz. $F_{T} S(n)$. Thus,

$$
V_{a b r}=k_{a b r} F_{T} S(n)=k_{a b r} \mu_{T} p A_{\mathrm{v}} \cos \theta[S(n)]
$$

To account for the influence of insert angle $\theta$ on wear severity, the abrasive wear rate is scaled by $\tan \theta$. When $\theta=45, \tan \theta=1.0$, based on defining $k_{45}$ as the volumetric abrasive wear rate of the surface at a 45 -degree seat angle, and in that case,

$$
V_{a b r}=\left(k_{45} \tan \theta\right)\left\{\mu_{T} p A_{\mathrm{v}} \cos \theta[S(n)]\right\}
$$

Considering the trigonometric identity $\tan \theta=(\sin \theta / \cos \theta)$, Eqn (14) reduces to

$$
V_{a b r}=k_{45} \mu_{T} p A_{\mathrm{v}}[S(n)] \sin \theta
$$

As the classical work on abrasion by Kruschov [12] and others has indicated, to first order, the abrasive wear rate of a metal is proportional to its hardness relative to the harder abradant, especially if there is at least a 20 percent difference in their hardness numbers [13]. Considering this, one might be tempted to scale Eqn. (16) by the relative hardness numbers of the valve and seat contacting materials; however, it is not that simple. After wear-in, the ACZ contains a mechanically-mixed tribolayer of metal and oxides, depending on the materials involved. Therefore, using the hot hardness numbers for the bulk valve and seat materials could be misleading. The abrasion to the valve and seat, in fact, will depend on the hardness of the tribolayer. If the layer adheres to one or both sides of the contact, the process could be 2-body abrasion against the opposite side, but if the 
tribolayer crumbles, there can be 3-body abrasion as well.

About hardness. Hardness is not included explicitly in Eqn (16), but rather is implicitly included in $k_{45}$. The abrasivity is determined by the nature of the material trapped between the contacting surfaces. To determine the magnitude of the abrasivity factor, more research is needed on threebody, high-temperature abrasive wear by oxide-metal mixtures, especially under repetitive impact with slip. Current experiments have shown how tribolayers similar in microstructure to those on valves can be generated in our laboratory $[4,7]$, but the results have not been sufficiently quantitative for use in the current model.

Abrasive wear of metals is caused by hard protuberances, and is affected by the load, and the tendency of a harder abradant to cut or to plow through the softer surface. Therefore, abrasive wear would be enhanced by protruding carbides on one or both materials or by the presence of hard thirdbodies in the interface. The latter could, for example, be wear particles or products of fuel combustion. It is known that one way to produce carbon nano-particles, including carbon nanotubes and fullerenes, is via combustion of carbonaceous compounds, so soot could contribute to the abrasion as well. Initially, the third-body concentration and the tendency for slip may be relatively low, but as the valve contact surface wears in and debris accumulates, the likelihood for slip may increase. This is reflected in the foregoing discussion (e.g., Fig. 102, 103). As for the deformation term, the net effects of abrasive wear can be expressed in terms of a projected area in the plane of the valve incline, by dividing by the circumference of the $\mathrm{ACZ}, \pi D_{\text {acz. }}$. Also, recognizing that for a valve presenting a circular face of diameter $d$ to the combustion chamber,

$$
\begin{aligned}
& A_{v}=\frac{\pi}{4} d^{2} \\
& A_{a b r}=\frac{V_{a b r}}{\pi D_{a c z}}
\end{aligned}
$$

Substituting for $V_{a b r}$, and defining $k^{*}$ as the abrasive wear rate at 45 seat angle, in terms of the projected area increase per unit normal force (i.e., $\mathrm{mm}^{2} / \mathrm{N}$ ), gives the following

$$
A_{a b r}=k * \mu_{T} p[S(n)] \sin \theta\left(\frac{d^{2}}{4 D_{a c z}}\right)
$$

Since, for a given valve design, $\theta, d$, and $D_{a c z}$ remain constant, Eqn. (19) becomes

$$
A_{\mathrm{abr}}=k^{*} \mu_{T} p c_{a 4}[S(n)]
$$

For example, when $\theta=45^{\circ}, d=42 \mathrm{~mm}$, and $D_{a c z}=38 \mathrm{~mm}$, the value of $c_{a 4}$ is $8.21 \mathrm{~mm}$. In terms of units, $\mu_{T}$ is dimensionless, $p$ is in $\mathrm{N} / \mathrm{mm}^{2}, S(n)$ is in $\mathrm{mm}, c_{a 4}$ is in $\mathrm{mm}$, and $k^{*}$ is in $\mathrm{mm}^{2} / \mathrm{N}$.

Values for $k^{*}$ for both valves and seats would reflect their individual characteristics; however, the sum of both contributions can be simplified. The value of $c_{a 4}$ is the same for both valve and seat surfaces because it depends on the geometry and dimensions of the given configuration. Likewise, the friction coefficient and maximum cylinder pressure are the same. Since there may be differences in the incubation period for the onset of abrasive wear on the two surfaces, the cumulative sliding distance during which abrasion occurs on valve and seat may not necessarily be equal. Thus, the total area on the profile that is due to the abrasive wear contribution after $\mathrm{n}$ cycles becomes: 


$$
\left.\left.A_{\mathrm{abr}}(n)\right]_{\mathrm{valve}}+A_{\mathrm{abr}}(n)\right]_{\mathrm{seat}}=\mu_{T} p c_{a 4}\left\{k^{*}{ }_{\text {valve }}[S(n)]_{\text {valve }}+k^{*}{ }_{\text {seat }}[S(n)]_{\text {seat }}\right\}
$$

\subsection{SUMMARY OF THE MODEL AND ITS FEATURES}

The model developed here is intended to compute the cross-sectional area on valve and seat due to wear and displacement of material. Thus, the abrasion and displacements of both valve and seat materials are added. The total area of the worn area $\left(A_{\mathrm{p}}\right)$ in a profile of the valve/seat combination after $n$ cycles is:

$$
\left.\left.A_{\mathrm{p}}(n)=A_{\mathrm{disp}}(n)\right]_{\mathrm{valve}}+A_{\text {disp }}(n)\right]_{\mathrm{seat}}+\mu_{T} p c_{a 4}\left\{k_{\text {valve }}[S(n)]_{\text {valve }}+k_{\text {seat }}[S(n)]_{\text {seat }}\right\}
$$

Table 1 summarizes the nomenclature and parameters within the terms of the model. The relatively large number of variables is a consequence of including adjustable rate constants to enable one to select the time needed to transition between the early and steady-state wear processes. One example of such a transition is running in. Other transitions may occur if prolonged exposure to temperature causes metallurgical changes to affect the properties of the materials, and still others are affected by the gradual evolution of a tribolayer in the interface.

To summarize, the model's main characteristics are as follows:

- The model consists of additive contributions from four terms: a displacement term and an abrasion term for the valve and for the mating seat.

- Each term can be separately modified to reflect system and materials characteristics.

- Recognizing that different forms of wear and surface damage can develop at different rates, there are incubation periods and transition rates built into each of the four terms.

- Constants reflect system characteristics, like the seat angle, and material characteristics, like the rate of work-hardening and its tendency to reach a saturation level beyond which further deformation is minimal.

- The friction coefficient appears in the model as a function of the operating temperature and helps account for the formation of lubricative oxides or tribolayers.

- The abrasive wear rate appears in the model referenced to a 45 degree seat angle.

- The abrasion term accounts for the effects of total sliding distance, which is affected by the amount of slip per cycle. The latter increases to a steady-state value after a period of wear-in. 
Table 1. Summary of Nomenclature Used in the Valve Wear Model

\begin{tabular}{|c|c|c|c|}
\hline $\begin{array}{l}\text { Applicable } \\
\text { Terms }\end{array}$ & Symbol & Description & Units \\
\hline \multirow[t]{4}{*}{ All } & $n$ & number of cycles & cycles \\
\hline & $d$ & diameter of the bottom face of the valve & $\mathrm{mm}$ \\
\hline & $D_{a c z}$ & average diameter of the annular contact zone on the valve & $\mathrm{mm}$ \\
\hline & $Z$ & axial direction along the valve stem & $\mathrm{mm}$ \\
\hline \multirow[t]{7}{*}{ Displacement } & $g(n)$ & volume displaced during the nth impact cycle & $\mathrm{mm}^{3}$ \\
\hline & $c_{d 1}$ & $\begin{array}{l}\text { change between the initial displacement and the steady-state } \\
\text { displacement }\end{array}$ & $\mathrm{mm}^{3}$ \\
\hline & $c_{d 2}$ & $\begin{array}{l}\text { rate constant reflecting how rapidly the displacement rate } \\
\text { reaches its steady-state value }\end{array}$ & $1 /$ cycles \\
\hline & $c_{d 3}$ & the intercept of the function $g(n)$ when $n=0$ & $\mathrm{~mm}^{3}$ \\
\hline & $C_{d 4}$ & deformation per cycle at steady-state (after wear-in) & $\mathrm{mm}^{3}$ \\
\hline & $V_{\text {disp }}$ & cumulative volume displaced after $n$ cycles & $\mathrm{mm}^{3}$ \\
\hline & $\mathrm{A}_{\text {disp }}$ & $\begin{array}{l}\text { projected area of displaced volume in the plane that contains } \\
\text { the valve seat incline }\end{array}$ & $\mathrm{mm}^{2}$ \\
\hline \multirow[t]{14}{*}{ Abrasion } & $c_{a 1}$ & half the magnitude of the slip length at steady-state & $\mathrm{mm}$ \\
\hline & $C_{a 2}$ & $\begin{array}{l}\text { rate constant for the transition from zero slip to the state-state } \\
\text { slip length }\end{array}$ & $1 /$ cycles \\
\hline & $C_{a 3}$ & $\begin{array}{l}\text { number of cycles to reach the mid-point of the transition } \\
\text { between zero slip and steady-state slip }\end{array}$ & cycles \\
\hline & $C_{a 4}$ & valve geometrical constant $=\left(\mathrm{d}^{2} / 4 \mathrm{D}_{\mathrm{acz}}\right) \sin \theta$ & $\mathrm{mm}$ \\
\hline & $S(n)$ & cumulative sliding distance & $\mathrm{mm}$ \\
\hline & $k_{45}$ & volumetric abrasive wear rate at 45 degrees seat angle & $\mathrm{mm}^{3} / \mathrm{N}$ \\
\hline & $k^{*}$ & $\begin{array}{l}\text { projected area (profile) abrasive wear rate of the surface at a } \\
\text { seat angle of } 45 \text { degrees }\end{array}$ & $\mathrm{mm}^{2} / \mathrm{N}$ \\
\hline & $p$ & $\begin{array}{l}\text { maximum combustion pressure in the cylinder (equates to } \\
\mathrm{MPa} \text { ) }\end{array}$ & $\mathrm{N} / \mathrm{mm}^{2}$ \\
\hline & $P_{n}$ & normal force on the sliding interface & $\mathrm{N}$ \\
\hline & $\theta$ & valve seat angle relative to the bottom face of the valve & deg \\
\hline & $\mu_{T}$ & $\begin{array}{l}\text { kinetic friction coefficient at the valve/seat interface at } \\
\text { temperature } \mathrm{T}\end{array}$ & (none) \\
\hline & $F_{T}$ & friction force on the valve/seat interface at temperature $\mathrm{T}$ & $\mathrm{N}$ \\
\hline & $V_{a b r}$ & volume abraded from a given surface & $\mathrm{mm}^{3}$ \\
\hline & $A_{a b r}$ & $\begin{array}{l}\text { projected area of the area of the valve or seat profile lost due } \\
\text { to abrasive wear }\end{array}$ & $\mathrm{mm}^{2}$ \\
\hline
\end{tabular}

Any tribological model, including the current one, includes a set of assumptions and simplifications. Sometimes these are stated and other times, they are implied. The key issue is whether the simplifying assumptions have a primary or secondary effect on the correlation between what is predicted and what is physically observed. Based upon observations like those shown in Figures 4-7, it is clear that the amount of wear is not the same everywhere in the annular valve and seat contact zone. Tribolayers or deformed metal may accumulate in some places more than in other places around the circumference. Tribolayers can cushion or protect surfaces from further wear temporarily, slowing the wear rate. The layers may also spall off from time to time, exposing the bare metal to further oxidation and wear. It is not unreasonable, therefore, that the terms in Eqn (22) may 
not operate continuously, but may 'turn on and off' over the lifetime of the valve/seat interface. One of the cases provided in Section 4 considers this possibility.

Before presenting some sample calculations using the current model, it will be instructive to compare its form with that of another recent model.

\subsection{COMPARISON WITH RECENT MODELS}

During the early 2000s, investigators at the University of Sheffield (UK) developed a model for valve recession $[14,15]$. The group, led by Dwyer-Joyce, noted that earlier valve wear models focused primarily on sliding contact, and that their new approach incorporated an impact component as well. The term-by-term derivation of the model by the Sheffield group is not repeated here; however, the model has the following form to compute the wear volume, $V$ :

$$
V=\left(\frac{k P_{a v e} N \delta}{h}+K N e^{n}\right)\left(\frac{A_{i}}{A}\right)^{j}
$$

where the variables in Eqn. (23) are listed in Table 2 for comparison to those in Table 1.

Table 2. Summary of Nomenclature Used in the Dwyer-Joyce Valve Recession Model

\begin{tabular}{|c|l|}
\hline Symbol & \\
\hline$k$ & non-dimensional sliding wear constant \\
\hline$P_{\text {ave }}$ & average load due to combustion \\
\hline$N$ & number of cycles of impact \\
\hline$\delta$ & slip distance in the interface \\
\hline$h$ & penetration hardness of the wearing surface \\
\hline$K$ & impact wear constant \\
\hline$e$ & impact energy per cycle $\left(1 / 2 \mathrm{mv}^{2}\right)$ \\
\hline$m$ & mass of the valve and follower added to half the mass of the spring \\
\hline$v$ & closing velocity at impact with the seat, as a function of the initial valve clearance \\
\hline$C_{i}$ & initial valve clearance \\
\hline$n$ & energy exponent for the impact wear term \\
\hline$A_{i} / A$ & ratio of the initial contact area to that after N cycles \\
\hline$j$ & empirical constant \\
\hline
\end{tabular}

The recession $(r)$ corresponding to a wear volume $V$ is computed from the seat insert angle $\theta$, the initial seat insert radius $\left(R_{\mathrm{i}}\right)$, and the measured initial seat face width $\left(w_{\mathrm{i}}\right)$ thus:

$$
r=\left[\left(\frac{V}{\pi R_{i} \cos \theta \sin \theta}+w_{i}^{2}\right)^{1 / 2}-w_{i}\right] \sin \theta
$$

The Dwyer-Joyce (D-J) model, which was later incorporated into a computer program called RECESS ${ }^{\mathrm{TM}}$, is composed of three stages. The sliding wear and impact terms are computed for $N$ cycles, added together, and then the recession is computed. This is similar to the current model which 
was developed independently, and actually was not compared to details of the D-J approach until it was completed. Some of the differences and similarities between these models may be summarized as follows:

- Both models are comprised of two contributions, one of which involves sliding (abrasion) and the other either impact or deformation. It can be argued that impact results in deformation, but deformation can also be due to tangential motions that produce a lip at one end of the contact.

- The D-J model implies an emphasis upon the least wear-resistant side of the contact coupon (presumably the valve), since there is only one sliding wear rate, one hardness number, and one impact wear term. Wear of the seat might be indirectly incorporated through the $\left(\mathrm{A}_{\mathrm{i}} / \mathrm{A}\right)$ ratio, and it might be argued that most of the recession is due to valve wear, but the current model does not presume this.

- The D-J model holds the slip distance constant, but the current model allows the slip distance to increase due to the evolution of wear.

- The current model provides for transitions in the wear rate directly via rate constants for each term, but the D-J model seems to accommodate them through an iterative process.

- The current model incorporates the effects of friction coefficient as function of temperature, but the D-J model does not treat those effects directly.

- The current model adjusts the relative contribution of sliding (abrasive) wear to deformation due to the influence of the seat angle, but the seat angle mainly enters into the D-J model in the final conversion of wear volume to recession.

- Both models contain empirical constants as well as physically-measurable quantities, so neither can fairly be called a 'first-principles' model.

\section{SAMPLE CALCULATIONS}

\subsection{CASE STUDIES}

Four versions of the valve wear model were run to examine the effects of various parameters on the change in the profile with number of cycles. In each version, one or more parameters were varied to investigate the effects they had on the total area of the worn profile. Before, exercising the model, it was necessary to know the friction coefficient as a function of temperature $\left(\mu_{T}\right)$. Rather than estimate these data, a high temperature pin-on-disk type tribometer was used to generate friction data at a series of sliding temperatures. That work is described in the following section.

\subsection{FRICTION EXPERIMENTS TO SUPPORT THE MODEL}

Experiments were performed using a high-temperature tribology test system (custom designed for ORNL by Advanced Mechanical Technology, Inc, Massachusetts). It is shown in Figure 14. For this experiment, an actual exhaust valve made from a Ni-based alloy called Pyromet $31^{\mathrm{TM}}$ was machined into a flat disk and the pin specimen was made from a Co-based alloy (Stellite $6 \mathrm{~B}^{\mathrm{TM}}$ ). The pin was spherically-ended with an initial radius of $9.53 \mathrm{~mm}$ and the test was conducted at a normal 
force of $10 \mathrm{~N}$ at a sliding speed of $0.1 \mathrm{~m} / \mathrm{s}$ in air. Sliding was done for 60 seconds at each of the following temperatures after first running-in the tip of the slider at room temperature for 60 seconds to wear it in: $450,550,650,750,800,850,800,750,650,550,450{ }^{\circ} \mathrm{C}$. In each case, a five minute soak was provided after each temperature was obtained. Sliding was stopped while the temperature was changing and reaching its next equilibrium.

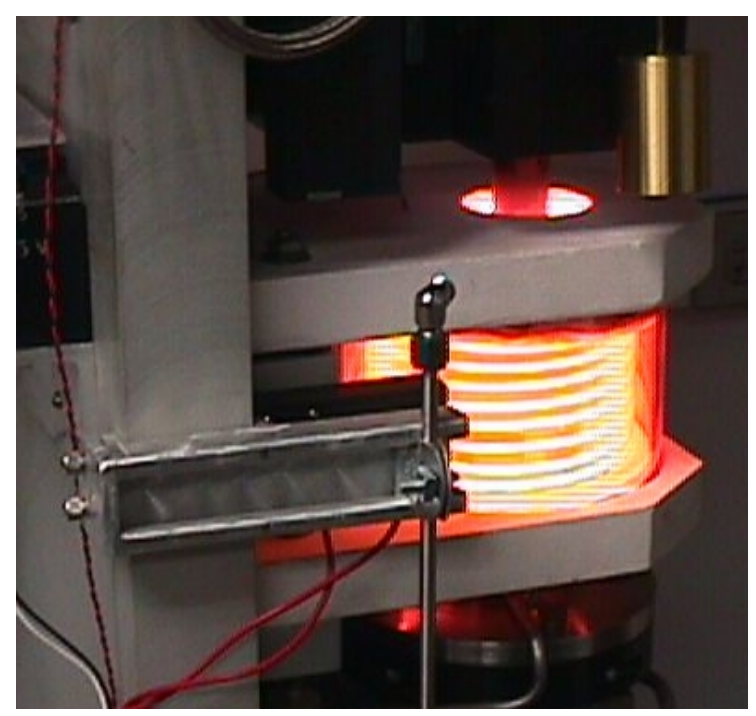

Figure 14. High-temperature pin-on-disk sliding friction and wear testing system. The pin specimen holder is visible at the top entering the furnace chamber where a disk rotates against it.

The friction coefficient at room temperature, during running-in, exhibited an average friction coefficient of 0.60 (std dev. 0.07). Results for elevated temperatures are given in Figure 15. With the exception of the tests at $750^{\circ} \mathrm{C}$, the lower of the two data at each temperature were for friction measured during the decreasing temperature stage of the experiment. The inset in the figure shows that the average friction coefficient at each temperature, over the temperature interval of $550-850{ }^{\circ} \mathrm{C}$, could be represented as a third-degree polynominal as follows:

$$
\mu_{T}=8.346-0.0347 T+4.90 \times 10^{-5} T^{2}-2.20 \times 10^{-8} T^{3}
$$

It was interesting to observe that the friction coefficient increased with rising temperature, despite the possibility that the oxide films that formed at high temperature could have acted as a lubricant. Apparently this was not the case. One might surmise that the observed rise in friction with temperature was a consequence of a higher 'plowing component' of the friction force that in essence overwhelmed the lubricative effects that oxides might have had in reducing 'the adhesive component' of friction, as described in the work of Bowden and Tabor [16]. The precise mechanistic reasons for this unexpected behavior remain to be determined; however, it is useful to note that the oxides that may have formed at the highest temperatures did not noticeably affect the friction as the temperature decreased again. 


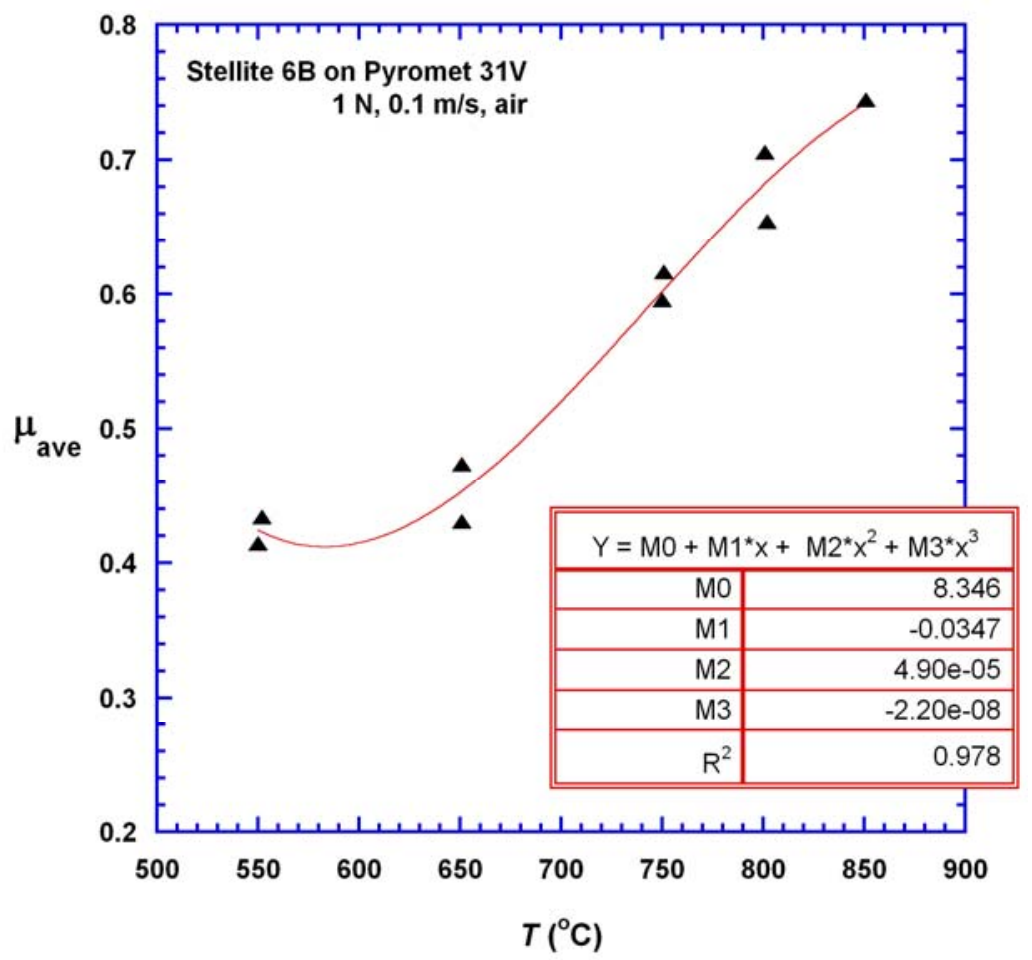

Figure 15. Sliding friction coefficient as a function of temperature for Stellite 6B pin on a rotating disk of Pyromet 31.

\subsection{RESULTS}

The values of the variables used in computing the four cases are summarized in Table 3 . The rationale for selecting those variables is explained in the following sections. Also, when selecting the magnitudes of the model's variables, the intent was to produce cross-sectional areas similar in magnitude to those observed on worn valves which were obtained through the courtesy of an engine manufacturer. Unfortunately, the exact engine operating time and average engine rpm was not known for the worn valves, so it was not possible to check the model predictions with measurements of the profiles on these valves. 
Table 3. Summary of Variables Used to Test the Valve Wear Model

\begin{tabular}{|c|c|c|c|c|c|c|}
\hline Term & Variable & Units & Case 1 & Case 2 & Case 3 & $\begin{array}{c}\text { Case } 4 \\
\text { (Note 2) }\end{array}$ \\
\hline \multirow[t]{7}{*}{ Common parameters } & $d$ & $\mathrm{~mm}$ & 42 & 42 & 42 & 42 \\
\hline & $D_{a c z}$ & $\mathrm{~mm}$ & 38 & 38 & 38 & 38 \\
\hline & $T$ & ${ }^{\circ} \mathrm{C}$ & 750 & 750 & 850 & 850 \\
\hline & $\begin{array}{c}\mu_{T} \\
\text { (Note 1) }\end{array}$ & (none) & 0.60 & 0.60 & 0.74 & 0.74 \\
\hline & $p$ & $\mathrm{MPa}$ & 18 & 18 & 18 & \\
\hline & $P_{n}$ & $\mathrm{~N}$ & $17,634$. & 17,634 . & 17,634 . & \\
\hline & $\theta$ & deg & 45 & 30 & 45 & 30 \\
\hline \multirow[t]{4}{*}{ Valve face displacement } & $c_{d 1}$ & $\mathrm{~mm}^{3}$ & 0.1 & 0.1 & $5 . \times 10^{-3}$ & 0.1 \\
\hline & $c_{d 2}$ & 1/cycles & $-1 . \times 10^{-5}$ & $-1 . \times 10^{-4}$ & $-1 . \times 10^{-4}$ & $-1 . \times 10^{-4}$ \\
\hline & $C_{d 3}$ & $\mathrm{~mm}^{3}$ & $-1 . \times 10^{4}$ & $-1 . \times 10^{4}$ & $-2.5 \times 10^{4}$ & $-2.5 \times 10^{4}$ \\
\hline & $C_{d 4}$ & $\mathrm{~mm}^{3}$ & 2. $\times 10^{-3}$ & 2. $\times 10^{-3}$ & 3. $\times 10^{-3}$ & $3 . \times 10^{-3}$ \\
\hline \multirow[t]{4}{*}{ Seat face displacement } & $C_{d 1}$ & $\mathrm{~mm}^{3}$ & - & $5 . \times 10^{-2}$ & $5 . \times 10^{-2}$ & - \\
\hline & $c_{d 2}$ & 1/cycles & - & $-5 . \times 10^{-3}$ & $-1 . \times 10^{-4}$ & - \\
\hline & $c_{d 3}$ & $\mathrm{~mm}^{3}$ & - & $-5 . \times 10^{3}$ & $-1 . \times 10^{4}$ & - \\
\hline & $c_{d 4}$ & $\mathrm{~mm}^{3}$ & - & 1. $\times 10^{-3}$ & 6. $\times 10^{-3}$ & - \\
\hline \multirow[t]{5}{*}{ Valve face abrasion } & $\overline{C_{a 1}}$ & $\mathrm{~mm}$ & 0.25 & 0.25 & .25 & .25 \\
\hline & $C_{a 2}$ & 1/cycles & 1. $\times 10^{-4}$ & 1. $\times 10^{-4}$ & 1. $\times 10^{-4}$ & 1. $\times 10^{-4}$ \\
\hline & $C_{a 3}$ & cycles & $2.5 \times 10^{4}$ & $2.5 \times 10^{4}$ & $5 . \times 10^{3}$ & 5. $\times 10^{3}$ \\
\hline & $C_{a 4}$ & $\mathrm{~mm}$ & 8.206 & 5.803 & 8.206 & 8.206 \\
\hline & $k^{*}$ & $\mathrm{~mm}^{2} / \mathrm{N}$ & 3. $\times 10^{-8}$ & 3. $\times 10^{-8}$ & $1 . \times 10^{-8}$ & 1. $\times 10^{-8}$ \\
\hline \multirow[t]{5}{*}{ Seat face abrasion } & $c_{a 1}$ & $\mathrm{~mm}$ & 0.25 & 0.25 & .25 & .25 \\
\hline & $C_{a 2}$ & 1/cycles & $5 . \times 10^{-5}$ & $5 . \times 10^{-5}$ & $5 . \times 10^{-5}$ & $5 . \times 10^{-5}$ \\
\hline & $c_{a 3}$ & cycles & 5. $\times 10^{4}$ & 5. $\times 10^{4}$ & 5. $\times 10^{4}$ & 5. $\times 10^{4}$ \\
\hline & $C_{a 4}$ & $\mathrm{~mm}$ & 8.206 & 5.803 & 8.206 & 8.206 \\
\hline & $k^{*}$ & $\mathrm{~mm}^{2} / \mathrm{N}$ & 1. $\times 10^{-8}$ & 1. $\times 10^{-8}$ & $5 \times 10^{-9}$ & $5 \times 10^{-9}$ \\
\hline
\end{tabular}

Notes:

(1) Friction coefficient was calculated for the given temperature from equation (21)

(2) In Case 4, the abrasion rate was temporarily interrupted (see the explanation of Case 4)

Case 1. This case assumes an exposure temperature of $750^{\circ} \mathrm{C}$ with a 45 degree seat angle and no contribution from the deformation of the seat. The abrasion rate of the seat was assumed to be $1 / 3$ that of the valve surface, and the incubation period for abrasion for the seat was twice that of the valve. The reason for the difference in incubation periods for seat and valve presume that the seat is more abrasion resistant than the valve and would only begin to abrade after the wear debris particles from the valve built up to a sufficient concentration in the tribolayer to begin to have an effect on the seat surface wear.

Figure 16 shows the form of the abrasive contribution alone, based on the parameters given in Table 1. There is only a subtle change in the slope of the total when the contribution of the seat wear becomes significant. If the seat wear rate were much higher, there would be a distinct kink in the total abrasive wear curve after the initiation period for the seat. In this example, the total crosssectional area due to abrasive wear after the first 100,000 cycles is about $2.5 \mathrm{~mm}^{2}$. This magnitude is not necessarily applicable to a specific engine, but serves as a baseline to enable the model to illustrate what changes might occur if a parameter such as insert angle or friction coefficient might 
change. For example, if the seat angle were only 30 degrees, the results would change to those in Figure 17, and the total wear becomes $1.7 \mathrm{~mm}^{2}$, a reduction of about $32 \%$.

The net effects of all four contributions to the model are shown in Figure 18. Contributions from abrasion and deformation are also shown in the figure. The effects of the incubation period are evident and the non-linear rate of profile area versus number of cycles is demonstrated.

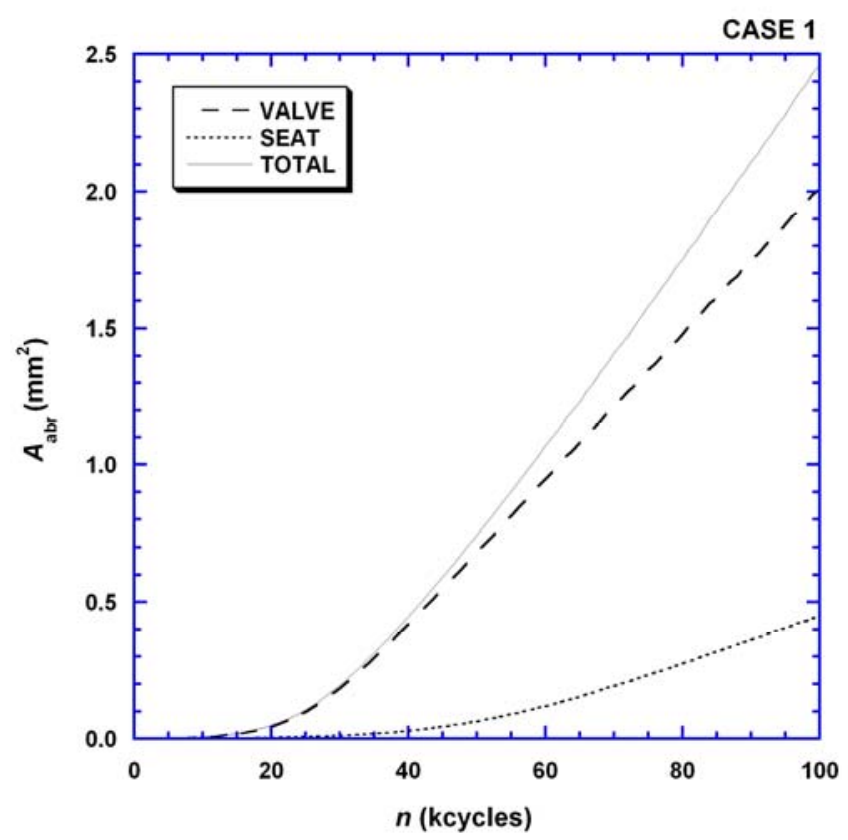

Figure 16. Area of the valve/seat profile due to the combined abrasive wear of both sides of the wear couple for Case 1.

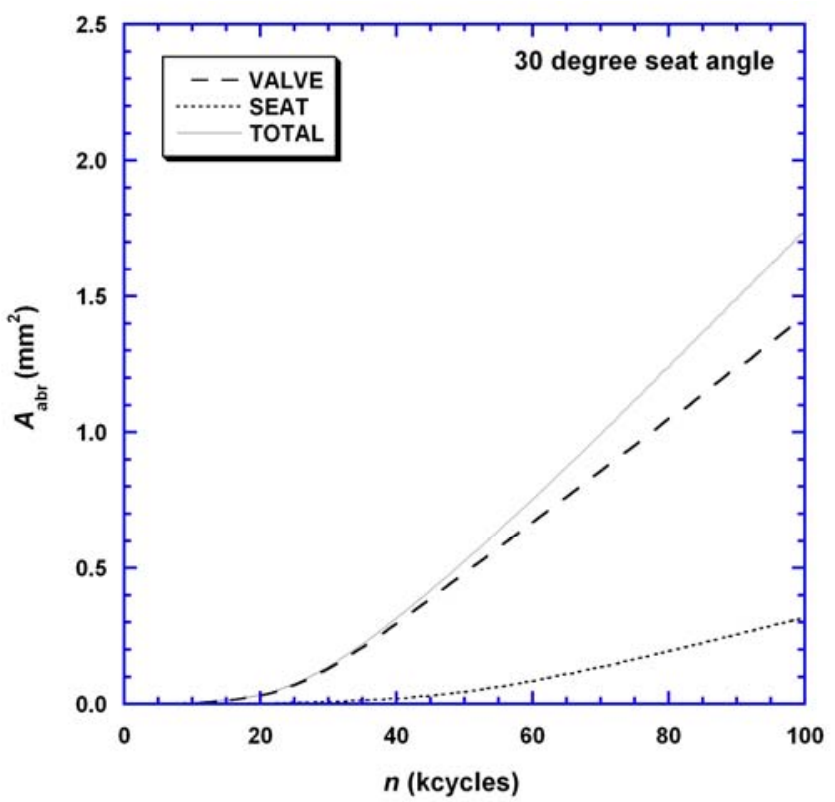

Figure 17. Area of the valve/seat profile due to the combined abrasive wear of both sides of the wear couple for Case 1 except that the seat angle is decreased to 30 degrees. 


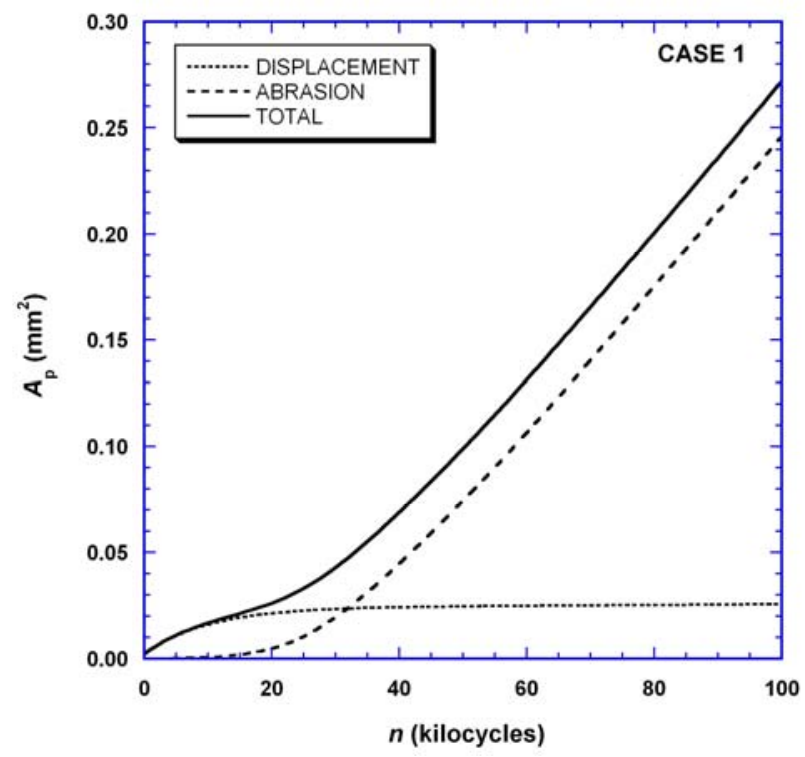

Figure 18. Plot of the area of the wear profile versus number of cycles of operation for the parameters applied in Case 1.

Case 2. The second demonstration case also assumes an exposure temperature of $750^{\circ} \mathrm{C}$, but with a shallower, 30 degree insert angle. The abrasion rates $k^{*}$ for the valve and seat were not changed from Case 1, but the effects of adding the deformation to the seat material were included. Results are shown in Figure 18. Since the seat angle is shallower in Case 2 than it is in Case 1, the effective abrasion contribution (slope of the abrasive contributions) is somewhat reduced, but that is to some extent compensated for by the increased contribution due to non-zero seat wear.

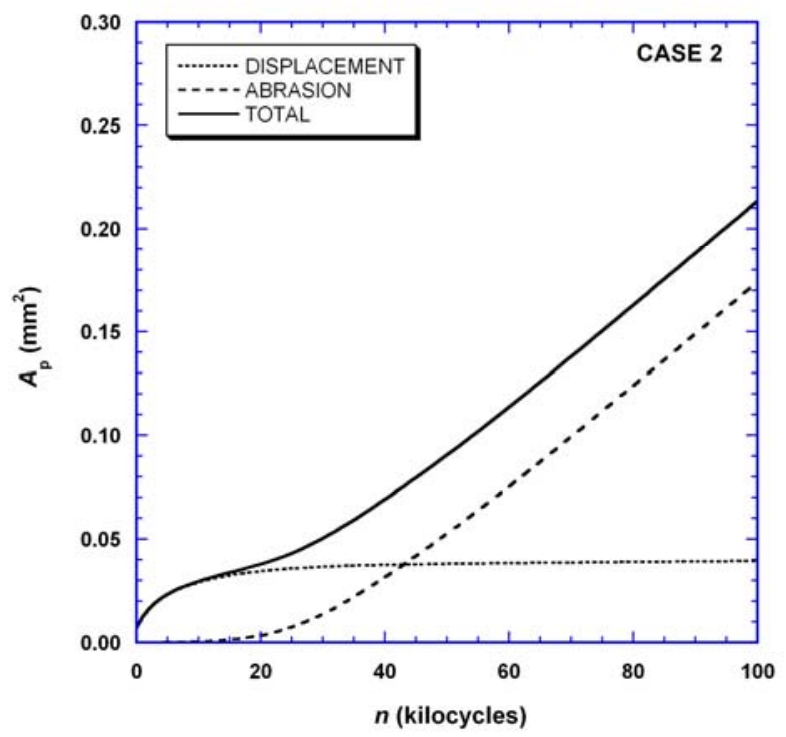

Figure 19. Plot of the area of the wear profile versus number of cycles of operation for the parameters applied in Case 2. 
Case 3. This case assumes an exposure temperature of $850^{\circ} \mathrm{C}$ with a 45 degree seat angle and longer incubation periods to reach the steady-state displacement rates for both the valve and seat surfaces. The friction coefficient is higher than for Cases 1 and 2. The relative abrasion rates of the two faces were decreased, assuming that the formation of a tribolayer at this higher temperature would retard the abrasive wear contribution. Note that the axes scales on Figure 20 are the same as those in Figures 18 and 19, allowing an easier comparison of the total profile area.

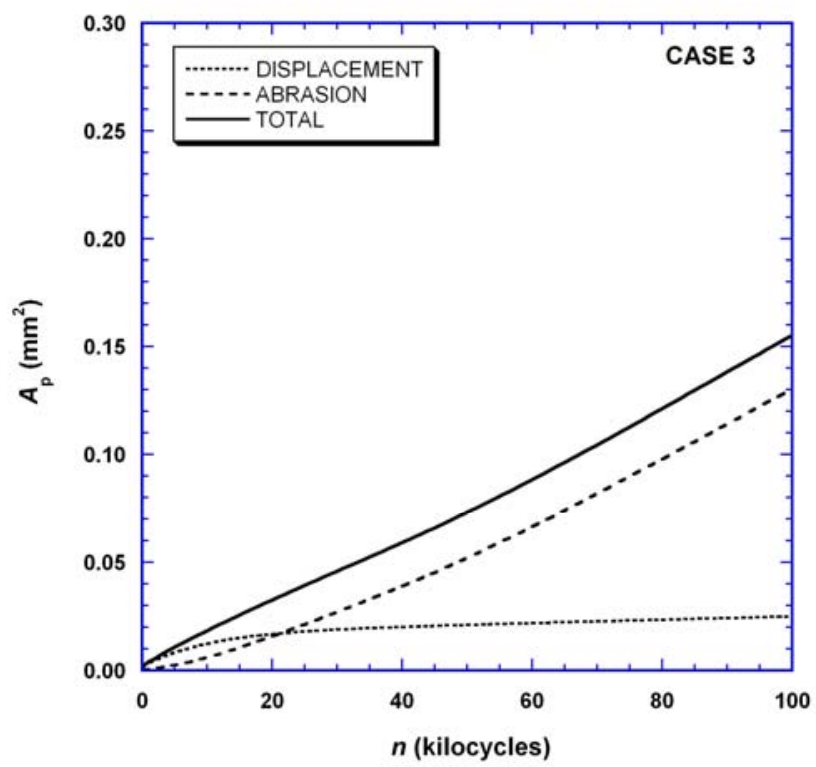

Figure 20. Plot of the area of the wear profile versus number of cycles of operation for the parameters applied in Case 3.

Case 4. This case portrays a hypothetical situation wherein a protective cushion of wear debris momentarily forms at 20,000 cycles and then is lost at 60,000, at which time the abrasion rates continue at the previous steady-state rates. Case 4 also assumes the same exposure temperature ( 850 ${ }^{\circ} \mathrm{C}$ ) and seat angle $\left(45^{\circ}\right)$ as for Case 3 , but with no displacement contribution from the seat material. As shown in Figure 21, initially, the material displacement on the valve dominates, then the abrasive wear contribution eventually becomes the dominant factor in the overall profile area generated by wear and surface damage.

The four cases described here exemplify the behavior of the model and its sensitivity to a number of possible scenarios, but the selected conditions did not account for the fact that in actual truck engines, the cylinder pressures, engine speed, and temperature cycles vary over the course of operation. Engines may idle for many hours or they make be called upon to labor up a steep hill or accelerate into busy traffic. Transients are likely to affect the wear of engine components, as much or more than the steady-state conditions do. 


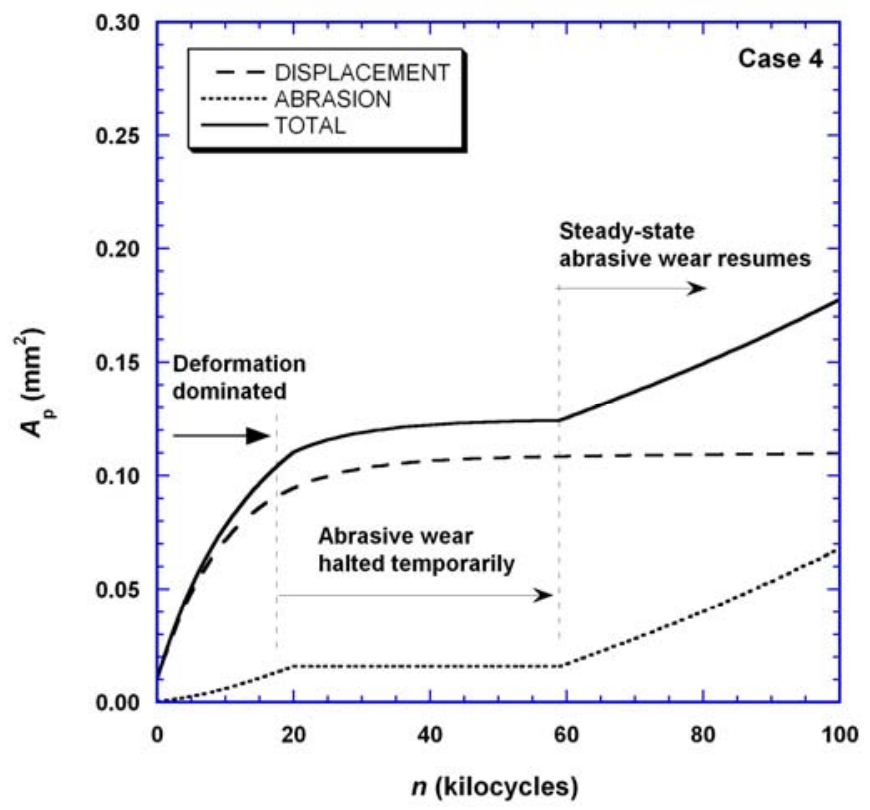

Figure 21. Representation of a situation in which the wear rate is interrupted by the formation of a protective tribolayer, then later resumes at its original steady-state rate.

Profile area and recession. From the cross-sectional area of the wear profile, it is possible to estimate the change in stem extension. If one assumes that the cross-sectional area is a rectangle of length $(L)$ equal to the contact length of the valve/seat interface and there is not significant extruded lip of material, then the depth of wear and deformation $(h)$ is $A_{p} / L$ (see Figure 22). Since the interface is inclined to the axis of the stem, the distance in stem extension due to wear and deformation is

$$
\Delta z=\left(\frac{A_{p}}{L}\right) \sec \theta
$$

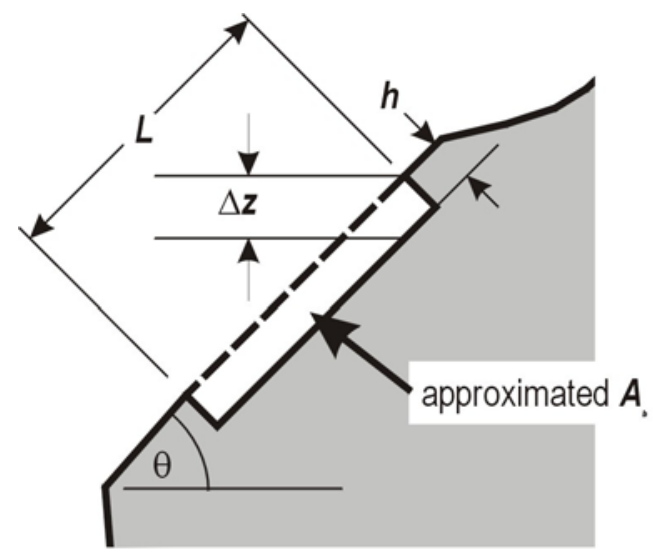

Figure 22. Approximation of the worn profile as a rectangle of length L. 
For the situation shown in Case 4, above, a plot of $\Delta z$ versus $n$ is shown in Figure 23, assuming a contact length $L=3 \mathrm{~mm}$. In this case, the deformation results in an initial depth of about 55-60 $\mu \mathrm{m}$, after which subsequent abrasion increases the depth to over $80 \mu \mathrm{m}$.

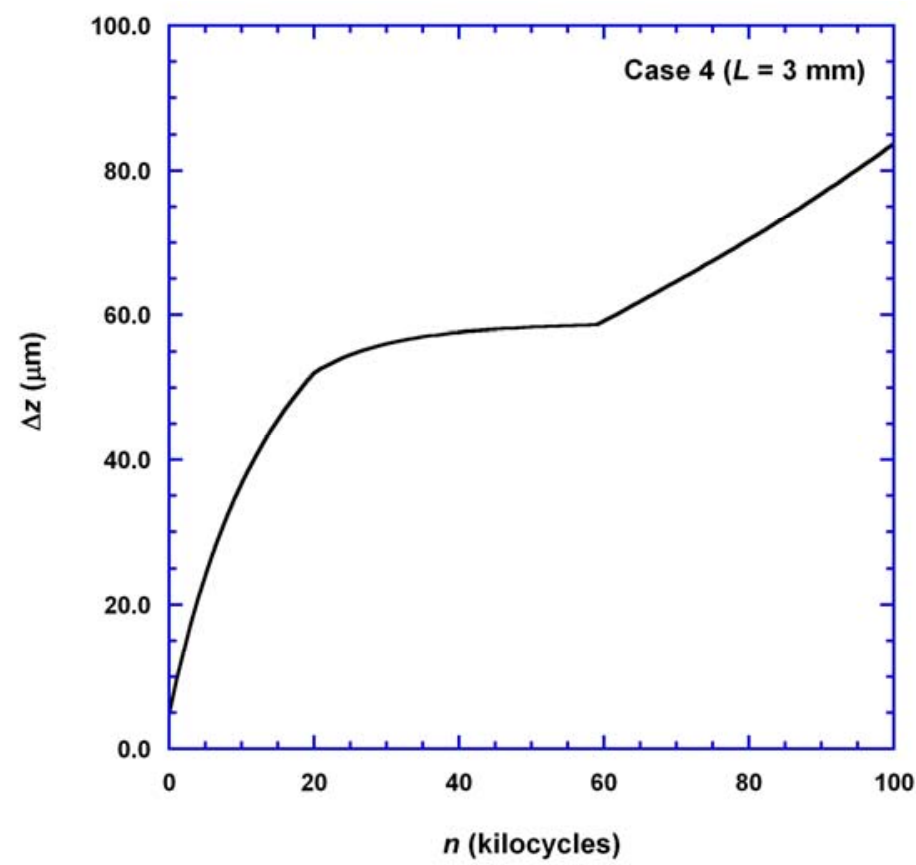

Figure 23. Estimation of valve stem recession from the model using Case 4 parameters.

\section{SUMMARY}

This report presents a mathematical model that portrays variations in the time-dependent removal and displacement of material from a diesel engine exhaust valve and seat combination. The model is based on the observation that valves experience a combination of repetitive impact with slip. Complications enter because tribochemical factors (oxidation and exposure to corrosive gases), alloy aging, and temperature cycles can alter the contact surface conditions over the lifetime of the components. Since the profile of wear can differ at different circumferential locations even on the same valve, it was impractical to model a generic wear profile, but rather to present results in terms of the cross-sectional area of material that has been lost in the valve-seat contact region from the conjoint action of deformation and abrasive wear. It is also recognized that tribolayers can form, and in effect add material to the profile (compared with wear processes that subtracts material from the profile). Observations of valve wear phenomena testify to the non-steady-state character of the process. They also suggest that commonly, much of the wear resulting in recession occurs on the valve sealing face rather than on the mating surface of the seat materials.

The model presented here consists of four terms to describe the cycle-dependent deformation 
rate and abrasion rate of the valve and the seat, respectively. In order to enable the rate of surface displacement and wear to vary during the initial wear-in process, a series of rate constants and incubation periods have been introduced. While these add to the number of adjustable parameters used in the model, it also increases the model's ability to account for such real-world occurrences as delayed initiation of the abrasive process and the decrease in the amount of deformation per impact cycle as the contact surfaces work-harden during use. Four examples were provided in this report to depict the change in the surface profile's cross-sectional area when different assumptions about the operating conditions are introduced. In one example, the abrasive wear process is halted temporarily to simulate the role of the formation of a protective oxide layer.

Most tribology bench tests, by nature, are conducted under steady-state conditions, rather than using the complex spectrum of loads, speeds, temperatures, and environments that occur during the course of a fired engine's lifetime. It is difficult to extrapolate steady-state results to predict valve wear life because the highest wear rates could occur during transient operation. An example of this phenomenon is the wear of aircraft brakes that is much higher during rejected take-off's than during normal taxiing and braking cycles. Similarly, it is recognized that wear of engines often is highest during cold start-up, when oil films have not yet been established. Friction experiments conducted in the course of this work indicate that the sliding resistance between surfaces, and the work deriving from it, varies with temperature as well. Therefore, additional parameters were introduced in the model in an attempt to account for the characteristics of transient processes.

Each engine, and in fact, even each cylinder in an engine, can experience different operating spectra. Therefore, selecting one valve and seat position to represent a given engine is not necessary representative of the 'average case.' However, in selecting materials for a valve/seat combination, one would tend to err on the side of simulating or modeling the most severe conditions. The parameters in the current model represent engine design aspects (for example, the seat angle), materials aspects (for example, the severity of deformation and the abrasive wear rate), and operating aspects (for example, the number of contact cycles, the slip per cycle, and the length of the running-in period). Unfortunately, the terms in the current model are not directly derivable from first principles of materials or mechanics, and that is an admitted short-coming. However, they do enable one to experiment with the effects of varying conditions to understand their collective influence on the timedependent wear response. 


\section{NUMBERED REFERENCES}

1. S. C. Davis and S. W. Diegel, Transportation Energy Data Book, $23^{\text {rd }}$ Ed., Oak Ridge National Laboratory report ORNL-6970, 2003.

2. Twentieth Century Truck Partnership: Roadmap and Technical White Papers, Report 21CT0003, U.S. Department of Energy, Energy Efficiency and Renewable Energy, Office of Vehicle Technologies, $72 \mathrm{pp}$. [online at http://www1.eere.energy.gov/vehiclesandfuels/pdfs/program/21ctp_roadmap_2007.pdf], 2006.

3. P. J. Blau and T. M. Brummett, High-Temperature Oxide Regrowth on Mechanically Damaged Surfaces" Tribology Letters, Vol. 32 (3), pp. 153-157, 2008.

4. P. J. Blau, T. M. Brummett, and B. A. Pint, Effects of Prior Surface Damage on HighTemperature Oxidation of Fe-, Ni-, and Co-Based Alloys, Wear, Vol. 267 (1-4), pp. 380-386, 2009.

5. P. J. Blau, Elevated Temperature Tribology of Metallic Alloys, pres. $2^{\text {nd }}$ Intl. Conf. on TriboCorrosion, Wiener Neustadt, Austria, March 17-18, 2009.

6 P. J. Blau and T. A. Hanft, Quantitative measurement of repetitive impact damage on ground silicon nitride surfaces, Tribology International, 27 (2), pp.109-118, 1994.

7. P. J. Blau, Development of a High-Temperature Repetitive-Impact Apparatus for Exhaust Valve Material Testing, paper no. IJTC2009-1503, extended abstract for the International Joint Tribology Conference, ASME/STLE, Memphis, TN, October 19-21, 2009.

8. N. Giles, Fundamentals of Valve Design and Material Selection, SAE Paper number 660471, Society of Automotive Engineers, Warrendale, Pennsylvania, 1996.

9. Y. S. Wang, S. Narasimhan, J. M. Larson, J. E. Larson, and G. C. Barber, The effect of operating conditions on heavy duty engine valve seat wear," Wear, 201, pp. 15-25, 1996.

10. S. K. Schaefer, J. M. Larson, L. F. Jenkins, and Y. Wang, Evolution of Heavy Duty Engine Valves, in Proc. Intern. Symposium on Valvetrain System Design and Materials, ASM International, Materials Park, OH, pp. 129-139, 1997.

11. N. Philips, Caterpillar, Inc., private communication, (July, 2009).

12. M. M. Kruschov, Proc. Conference on Lubrication and Wear, Institute of Mech. Engr., p. 655, 1957.

13. D. Tabor, The Hardness of Metals, Oxford Press, Oxford, UK, 1951.

14. R. Lewis and R. S. Dwyer-Joyce, Design Tools for Prediction of Valve Recession and Solving Valve/Seat Failure Problems, SAE Paper 2001-01-1987, Soc. of Automotive Engineers, Warrendale, PA, 10 pp., 2001. (also printed in SAE Spec. Publication SP-1624)

15. T. Slatter, R. Lewis, and R. S. Dwyer-Joyce, Valve Recession Modelling, SAE Paper 2006-010365, Soc. of Automotive Engineers, Warrendale, PA, 7 pp., 2006. 
16. F. P. Bowden and D. Tabor, TheFriction and Lubrication of Solids, Oxford Press, UK, 1950.

\section{ANNOTATED BIBLIOGRAPHY ON VALVE WEAR}

The following references concern the mechanisms of wear in internal combustion engine valves, materials selection for valves, and the effects of engine operating conditions on valve durability. They are listed chronologically, by year of publication.

1) E. F. de Wilde (1967) “Investigation of Engine Exhaust Valve Wear," Wear, Vol. 10, pp. 231-244. Investigation of exhaust valve wear in a spark ignition engine. Involved measurements of valve and seat temperatures, a dynamic analysis, and metallurgical studies of the contact surfaces. Concluded that reduction in the friction coefficient was important to reduce the wear.

2) S. L. Narasimhan, J. M. Larson, and E. P. Whelan (1981-82) "Wear characterization of new nickel-base alloys for internal combustion engine valve SEAT applications," Wear, Vol. 74, pp. 213227.

Emphasis was on identifying viable candidate Ni-base alloys to replace Co-based alloys. Discussed wear modes including adhesive, abrasive, and corrosive/erosive wear (guttering). Designed a valve/seat test system and conducted experiments at $425^{\circ} \mathrm{C}$. Considered hot hardness and several types of bench-scale wear test results. Concluded that there were viable Ni-based alloys to replace Co-based alloys for valves.

3) M. J. Malatesta, G. C. Barber, J. M. Larson, and S. L. Narasimhan (1993) "Development of a Laboratory Bench Test to Simulate Seat Wear of Engine Poppet Valves," Tribology Trans., Vol. 35, pp. 627-632.

Describes the construction and use of a vertical, heated single valve test apparatus. Used a variety of loads from $15-21 \mathrm{kN}$, various seat offsets, and a typical valve test temperature of $538^{\circ} \mathrm{C}$ and $166-324^{\circ}$ $\mathrm{C}$ for the seat. Measured wear depth as well as the material displaced in the lip at the edge of the contact area. Valve-insert misalignment was seen as a major contributor to the wear.

4) Y. S. Wang, S. K. Schaefer, C. Bennett, and G. C. Barber (1995) „Wear Mechanisms of Valve Seat and Insert in Heavy Duty Diesel Engine," SAE paper 952476, Soc. of Automotive Engineers, Warrendale, PA. 11 pp. (also printed in SAE Spec. Publication SP-1116).

Indicated the range of peak combustion pressures and temperatures for valve faces and seats. Studied 164 valves from 47 field engines. The dominant wear mechanisms and forms of surface damage were seen to vary due to operating conditions. Shear strain on the surfaces was found to be important from a wear standpoint, suggesting a strategy of reducing the friction coefficient. High combustion pressure tends to promote adhesive wear, and facing valves with Co-based alloys helped alleviate this problem. Alignment was seen to affect contact stresses and modes of damage. Sources of abrasive wear were discussed. Corrosion-assisted wear was important in some cases.

5) Y. S. Wang, S. Narasimhan, J. M. Larsen, J. E. Larson, and G. C. Barber (1996) "The effect of operating conditions on heavy duty engine valve seat wear," Wear, Vol. 201, pp. 15-25.

A heated valve test rig, described in previous papers, was used to conduct studies of the effects of various loads $(6.6-24.3 \mathrm{kN})$, numbers of cycles, and temperatures $\left(180\right.$ to $\left.650^{\circ} \mathrm{C}\right)$ on the wear of two fe-based alloys Sil 1 and Sil XB. There was an exponential relationship of wear with time due to the increase in contact area from wear-in. Wear scar size (depth) decreased at higher temperatures $(>$ $400^{\circ} \mathrm{C}$ ) due to oxide layer formation. Therefore, two different wear regimes were identified 
depending on temperature.

6) H. A. Bolton and J. M. Larsen, ed. (1997) Valvetrain System Design and Materials, ASM International, Materials Park, Ohio, $139 \mathrm{pp}$.

Conference proceedings containing 13 papers from a conference held in April 1997. Two papers of special interest in this project are listed separately, below.

7) D. Dooley, T. Trudeau, and D. Bancroft (1997) "Materials and Design Aspects of Modern Valve Seat Inserts," published in ref (6), pp. 55-63.

Discusses various seat insert profiles and their advantages and disadvantages, then describes the metallurgical aspects of seat materials, including both ferrous and non-ferrous alloys processed by several routes such as casting, powder forging, powder metallurgy, and liquid phase sintering.

8) S. K. Schaefer, J. M. Larsen, L. F. Jenkins, and Y. Wang (1997) "Evolution of Heavy Duty Engine Valves - Materials and Design," published in ref (6), pp. 129-139.

Describes materials requirements, including the role of corrosion and the temperature distributions on valves, and the effects of stricter emissions requirements and engine designs on the selection of valve and seat materials. Discusses evolution of alloy compositions and properties for intake and exhaust valves as well as seats. Lists material compositions for heavy duty engine intake valves, exhaust valves, and seats.

9) R. Lewis, R. S. Dwyer-Joyce, and G. Josey (1999) "Investigation of Wear Mechanisms Occurring in Passenger Car Diesel Engine Inlet Valve and Seat Inserts," SAE Paper 1999-01-1216, Soc. of Automotive Engineers, Warrendale, PA, 9 pp.

Describes the design of a hydraulically-driven, bench-top type valve testing apparatus that permits a variety of operating variables, including misalignment and circumferential sliding (induced rotation), to be investigated. Discusses the roles of impact plus sliding wear.

10) X. Liang, G. Strong, D. Eickmeyer, and K. Myers (1999) "A Study of Valve Seat Insert Wear Mechanisms," SAE Paper 1999-01-3673, Soc. of Automotive Engineers, Warrendale, PA, 12 pp. Investigated the wear of 10 valve seat insert materials, including both commercial and experimental alloys. Six were iron-based, two were nickel based, and two were cobalt-based. A series of micrographs of surfaces and cross-sections are shown. Wear depends partly on the presence of hard phases in the microstructure, but hardness is not the sole wear-controlling characteristic. There is an extended discussion on the role of oxidation, adhesion, and ductility on insert wear mechanisms in $\mathrm{Fe}$, $\mathrm{Ni}$, and Co based alloys. The formation and removal of tribolayers is also discussed.

11) R. Lewis and R. S. Dwyer-Joyce (2001) "Design Tools for Prediction of Valve Recession and Solving Valve/Seat Failure Problems," SAE Paper 2001-01-1987, Soc. of Automotive Engineers, Warrendale, PA, 10 pp. (also printed in SAE Spec. Publication SP-1624)

Provides a derivation for a model for valve wear that was later enhanced (see Ref 14, below). The two primary contributions were impact and micro-sliding. Modeled the effects of impact using an expression adapted from erosive wear studies. Estimates the relative contributions of impact and sliding.

12) G. Roth (2003) "Fatigue Analysis Methodology for Predicting Engine Valve Life," SAE Paper 2003-01-0726, Soc. of Automotive Engineers, Warrendale, PA, 11 pp.

Elaborates on the details of a study of finite element modeling to accommodate the influences of a variety of variables on the growth of fatigue cracks, mainly in the region at the base of the stem. 
Discusses thermal distortion analysis, combustion loads, assembly tolerances, seat misalignments, closing impacts, and cumulative fatigue damage.

13) K. J. Chun, J. S. Hong, H. J. Lee, D. Y. Kim, and J. K. Im (2004) "A Study on Engine Valve and Seat Insert Wearing Depending on Speed Change," SAE Paper 2004-01-1655, Soc. of Automotive Engineers, Warrendale, PA, 7 pp.

A valve and seat bench-scale testing system is described. The rate of valve wear and the surface roughness of the valves and seats were affected by speed. At lower speeds, adhesive wear was more prominent, but as speed increased, abrasive wear contributions became more important. (Note that the model described in this report assumes that abrasive wear is operating.)

14) T. Slatter, R. Lewis, and R. S. Dwyer-Joyce (2006) "Valve Recession Modelling," SAE Paper 2006-01-0365, Soc. of Automotive Engineers, Warrendale, PA, 7 pp.

Describes the rationale for a valve wear model and presents block diagrams to describe the operation of a computer program named RECESS. Provides results of several experimental validation studies using motorized cylinder heads. Discusses predictions of the relative role of sliding wear in valve recession. This can vary from $2-30 \%$ of the wear contribution, depending on operating conditions.

15) K. J. Chun, J. H. Lim, and J. S. Hong (2007) "A study of exhaust valve and seat insert wear depending on cycle numbers," Wear, Vol. 263, pp. 1147-1157.

Describes a dedicated valve wear testing machine and how it was used to generate wear damage to study the mechanisms of seat and valve wear. The distance between the maximum and minimum features on the worn area was used as a criterion for wear measurement. Micrographs and surface chemical analysis of tribochemical reaction products are provided. The discussion includes a proposal for the generation, compression, and movement of debris particles within the contact interface.

16) Y. Wang (2007) Introduction to Engine Valvetrains, book, SAE International, Warrendale, PA. $585 \mathrm{pp}$.

Monograph describing all aspects of engine valve trains, including chapters on valvetrain tribology and failure analysis. A table in Chapter 6 lists the various valvetrain components and the forms of wear and surface damage that occurs 


\section{General Nomenclature*}

BSE

D-J model

friction coefficient

HTRI

tribolayer
Back-scattered electron image obtained with a scanning electron microscope Valve wear model developed by the group of Dwyer-Joyce at the University of Sheffield (this report, references 11 and 14) ratio of the force resisting relative, tangential motion between two bodies to the force normal to the interface between them High-temperature, repetitive impact testing system designed for this project

A mixture of materials formed in an interface due to rubbing contact

* In addition, see Table 2 for the definition of symbols used in the model. 


\section{INTERNAL DISTRIBUTION}

1-3. D.R. Johnson, 4515, MS-6066

7-10. P.J. Blau, 4515, MS-6063

11. J. Qu , 4515, MS-6063

12. B.A. Pint, 4500 S, MS-6156

13. B.G. Bunting, NTRC, MS-6472

14. R.L. Graves, NTRC, MS-6472

15. H.T. Lin, 4515 , MS-6068

16. R.G. Boeman, NTRC, MS-6472

17. Lab Records, 4500 N, MS-625

18. ORNL Central Research Library, 4500N, MS-6191

19. ORNL Office of Technical Information and Classification

\section{EXTERNAL DISTRIBUTION}

20-25. Jerry Gibbs, Vehicle Technologies Program, U.S. Dept. of Energy, EE-2G, 1000 Independence Avenue SW, Washington, DC 20585

26. Nate Phillips, Caterpillar, Inc., 4009 Old Galena Road, Mossville, IL 61552

27. Randy Stafford, Cummins Engine Company, Box 3005, Columbus, IN 47202-3005. 
